\title{
EchoGéo
}

47| 2019

Nouvelles géographies de la collecte

\section{La mise en image du rebut}

Matières, corp(us) et pratiques autour des déchets

\section{Claudia Cirelli, Bénédicte Florin et Rémi de Bercegol}

\section{OpenEdition}

\section{Journals}

Édition électronique

URL : https://journals.openedition.org/echogeo/17103

DOI : $10.4000 /$ echogeo.17103

ISSN : 1963-1197

Éditeur

Pôle de recherche pour l'organisation et la diffusion de l'information géographique (CNRS UMR 8586)

Référence électronique

Claudia Cirelli, Bénédicte Florin et Rémi de Bercegol, « La mise en image du rebut », EchoGéo [En ligne], 47 | 2019, mis en ligne le 21 avril 2019, consulté le 10 août 2021. URL : http://journals.openedition.org/ echogeo/17103; DOI : https://doi.org/10.4000/echogeo.17103

Ce document a été généré automatiquement le 10 août 2021.

EchoGéo est mis à disposition selon les termes de la licence Creative Commons Attribution - Pas d'Utilisation Commerciale - Pas de Modification 4.0 International (CC BY-NC-ND) 


\title{
La mise en image du rebut
}

\author{
Matières, corp(us) et pratiques autour des déchets \\ Claudia Cirelli, Bénédicte Florin et Rémi de Bercegol
}

\author{
Photographies et légendes des photographies : \\ Rémi de Bercegol, chargé de recherche au CNRS et membre de l'UMR PRODIG, \\ Jérémy Cavé, consultant indépendant en écologie urbaine et enseignant à l'IEP, \\ Claudia Cirelli, chercheure associée de l'UMR CITERES, \\ Bénédicte Florin, maitre de conférences à l'Université de Tours et membre de l'UMR CITERES, \\ Pascal Garret, architecte, sociologue et photographe indépendant \\ Mikaëla Le Meur, doctorante à l'Université Libre de Bruxelles, \\ Adeline Pierrat, maitre de conférences en géographie à l'Université du Mans et membre de \\ l'UMR ESO, \\ Mélanie Rateau, doctorante au LATTS, \\ Rémi Reboux, diplômé à l'EHESS Marseille et à Sciences Po Paris.
}

\section{Introduction}

1 Figures marginales, les individus qui ont affaire aux rebuts tendent à être identifiés à la matière de leur travail. Si l'acte de récupérer permet de sauver le déchet de la disparition grâce au réemploi et au recyclage, tout aussitôt, il corrompt et stigmatise celui qui le manipule (Lhuilier et Cochin 1999). Cette superposition est illustrée, volontairement ou non, par les nombreuses images mettant en scène des femmes et des hommes travaillant dans des conditions dégradantes, peuplant des paysages dantesques, évoquant et figeant une misère humaine dont ils seraient l'un des emblèmes.

2 Il en est tout autrement pour les photographies documentées dans ce portfolio qui ont pour ambition d'exposer sous un autre jour l'univers des «travailleurs des déchets » (Corteel et Le Lay 2011). Extraites de l'exposition itinérante La mise en image du rebut: matières, corp(us) et pratiques autour des déchets ${ }^{1}$, ces photographies résultent d'une réflexion sur le statut des images dans les travaux de recherches sur les déchets. Il s'agit d'un travail mis en place par les chercheurs du réseau Sociétés urbaines et déchets 
(SUD $)^{2}$ qui, à partir d'approches et de terrains divers, analyse les processus d'ordre social, culturel, politique, économique et spatial qui se déploient autour de la gestion des déchets et qui touchent ces travailleurs. Les modalités de construction de ce corpus photographique ont fait initialement l'objet d'un séminaire-atelier pluridisciplinaire en 2016 rassemblant des membres du réseau SUD et des chercheurs extérieurs spécialistes de l'image (photographie et anthropologie, cinéma ethnographique, commissaires d'exposition). Les débats ont porté sur la place des images dans les recherches scientifiques, sur le pouvoir et les valeurs que ces images véhiculent ainsi que sur leur réception sociale. À l'instar de Daniel Friedmann (2010), qui préconisait pour le film l'analyse des effets socio-filmiques, nous avons questionné les effets sociophotographiques de cette mise en image au-delà de ce que les images mettent en scène. Comment les images captent-elles et traduisent les mutations sociales dont les déchets font l'objet ? Qu'est-ce que cette « mise en image sociale » (Gunthert, 2007) exprime et révèle de l'évolution de la place des travailleurs des déchets dans nos sociétés urbaines? Les enjeux relatifs à une "noblesse de l'abject " (Dagognet, 2009) et à une "esthétique du désordre" (Jeanjean, 2006) ont également été discutés: les photographies visent-elles uniquement à enrichir, voire simplement " embellir " (Sontag, 1977), le propos écrit? Ou bien constituent-elles un matériau, un outil, un langage, spécifiques qui, en rendant visible ces travailleurs marginalisés, permettraient de « découvrir des aspects du réel que la rapidité du mouvement de la vie avait tenus cachés » (Louveau de la Guigneraye et Arlaud, 2007, p. 104)?

Ce sont ici quelques-unes des questions qui continuent à alimenter l'évolution de l'exposition, enrichie peu à peu par de nouvelles images sélectionnées selon un ensemble de critères éthiques (sur la dignité, le droit à l'image, le consentement du sujet), contextualisées sociologiquement par un entretien systématique (du chercheur avec les personnes photographiés) et renseignées par des éléments techniques sur la prise de vue (appareil, lieu, date) ${ }^{3}$.

4 Loin de renvoyer au public une image de la misère et de la marginalité, ces portraits de récupérateurs souhaitent mettre en lumière des femmes et des hommes débarrassés du stigmate qui accompagne le contact avec l'ordure. Il s'agit ici de travailleurs normaux, occupés à réaliser leurs tâches; des travailleurs qui désirent être considérés comme égaux des autres, reconnus dans leur travail et par la société. De plus en plus, ils revendiquent des droits sociaux, mais aussi la légitimité de leur contribution au recyclage, à la gestion des déchets et, plus globalement, à la protection de l'environnement. Les poses qu'ils prennent spontanément à la demande du chercheur, ici aussi photographe, ainsi que les sourires et les regards destinés à l'objectif témoignent de cette aspiration à donner d'eux-mêmes et de leur activité un portrait ordinaire contribuant par cette mise en scène à une reconnaissance et à une requalification de leur métier et, de ce fait, de leur image.

5 Ce portfolio, présentant une courte sélection du corpus de l'exposition, est structuré en trois temps qui correspondent aux trois principales phases de la valorisation des déchets : la collecte en ville ou dans les décharges, puis le travail de tri, démontage, nettoyage des matériaux effectués dans les quartiers des récupérateurs, les dépôts ou les entrepôts des coopératives, et, enfin, la phase de transformation où s'opère la transmutation des matériaux avant leur commercialisation. 


\section{La collecte}

6 La série de photographies sur la collecte en ville souhaite mettre en lumière les pratiques (diurnes ou nocturnes), l'inventivité quant aux outils et véhicules utilisés, les parcours des récupérateurs, mais aussi leur forte connaissance de l'espace urbain: les portraits présentés ci-dessous montrent qu'ils savent où et quand trouver la ressource la plus valorisable en fonction des quartiers, des rues et parfois même à l'échelle de l'immeuble. Leur savoir-faire se déploie dans les espaces publics où ils sont particulièrement repérables, et souvent vulnérables, ce qui les oblige à "négocier » leur présence avec les habitants, les autorités, la circulation.

7 La collecte s'effectue aussi à même les décharges qui coïncident, dans certains cas, avec le lieu d'habitat. Sur les décharges, le méthane, lié à la fermentation, forme parfois des poches de gaz hautement inflammable et explosif, pouvant engloutir les travailleurs et provoquer des incendies. Les éboulements de terrains ou affaissement des monticules de déchets restent fréquents, tel l'accident mortel survenu sur la décharge d'AddisAbeba en mars 2017 ou sur celle de Ghazipur à Delhi en septembre de la même année. Le « jus » - appelé lixiviat - des déchets est chargé de polluants organiques, chimiques et de métaux lourds. Ces dangers se superposent aux risques sanitaires, maladies et blessures aux incidences beaucoup plus élevées qu'ailleurs (Chokhandre et al., 2017).

Dans les quartiers où se superposent espace de vie et espace de travail, la proximité avec les déchets affecte aussi l'environnement des récupérateurs: accumulation des résidus en attente de traitement; déchets irrécupérables qui ne sont parfois jamais évacués mais peuvent être brûlés par les récupérateurs eux-mêmes afin d'en réduire la quantité ou pour en extraire des matériaux comme le cuivre par exemple; pollution atmosphérique, des sols, des cours d'eau et des nappes superficielles...

9 Comme en témoignent les images, une autre modalité de la "récupération informelle » est réalisée par les employés municipaux chargés de la collecte des déchets, par exemple à Mexico ou à Lima. Cela consiste à sélectionner et à trier les matériaux intéressants avant qu'ils n'atterrissent dans la benne à ordures (ou le dhalao - point de collecte - à Delhi).

10 Sans jamais oublier les contextes spécifiques liés aux mondes des déchets, ni atténuer les risques encourus par ceux qui « font avec », les portraits ci-dessous, appuyés sur des entretiens menés par les chercheurs, révèlent néanmoins que le travail réalisé par ces femmes et hommes est un maillon essentiel de la chaîne de collecte des déchets et, plus largement, d'un système qui ne pourrait fonctionner sans eux. 
Illustration 1 - Crochets et paniers : les outils des récupérateurs

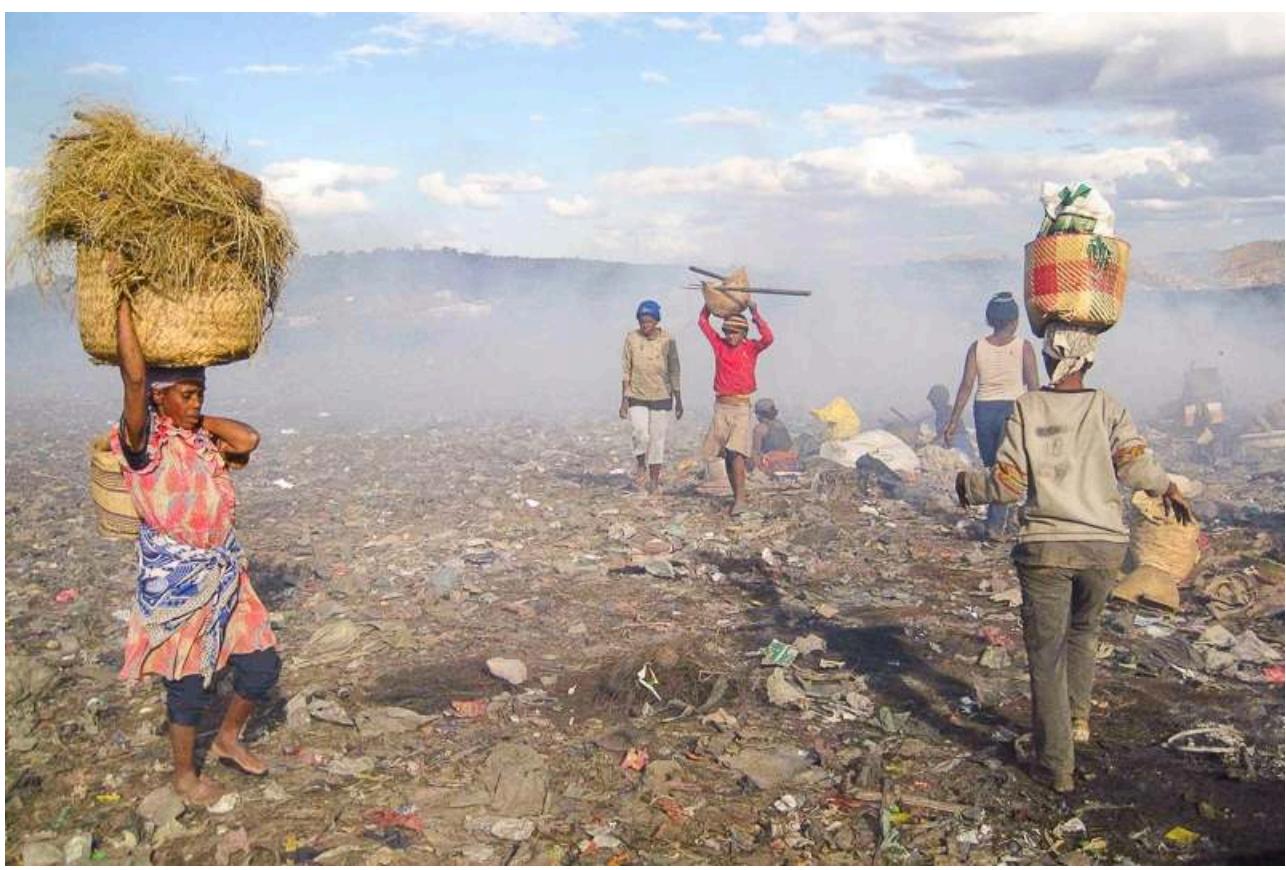

Cette photographie illustre le mouvement, plutôt lent, des récupérateurs dans la décharge d'Andralanitra, alors qu'ils terminent leur travail en milieu de matinée. C'est la nuit que ces travailleurs, appelés « chiffonniers » à Madagascar, fouillent la montagne d'ordures pour en extraire les objets les plus lucratifs qui arrivent sur le site. Les paniers et les crochets que l'on aperçoit ici sont les seuls outils dont ils disposent pour travailler. Ils constituent le premier maillon d'un réseau complexe d'acteurs intermédiaires. Ces matériaux et objets sont ensuite vendus à des grossistes, destinés à des marchés spécialisés du centre-ville ou à des ateliers d'artisans. Les chiffonniers d'Andralanitra ne montrent pas d'hostilité vis-à-vis du photographe. Cette attitude est liée à la médiatisation de l'action menée depuis 1989 par le Père Pedro et son association caritative auprès des plus démunis.

Antananarivo, Madagascar, juin 2015. Auteur : A. Pierrat. 
Illustration 2 - Tirer sa force des déchets

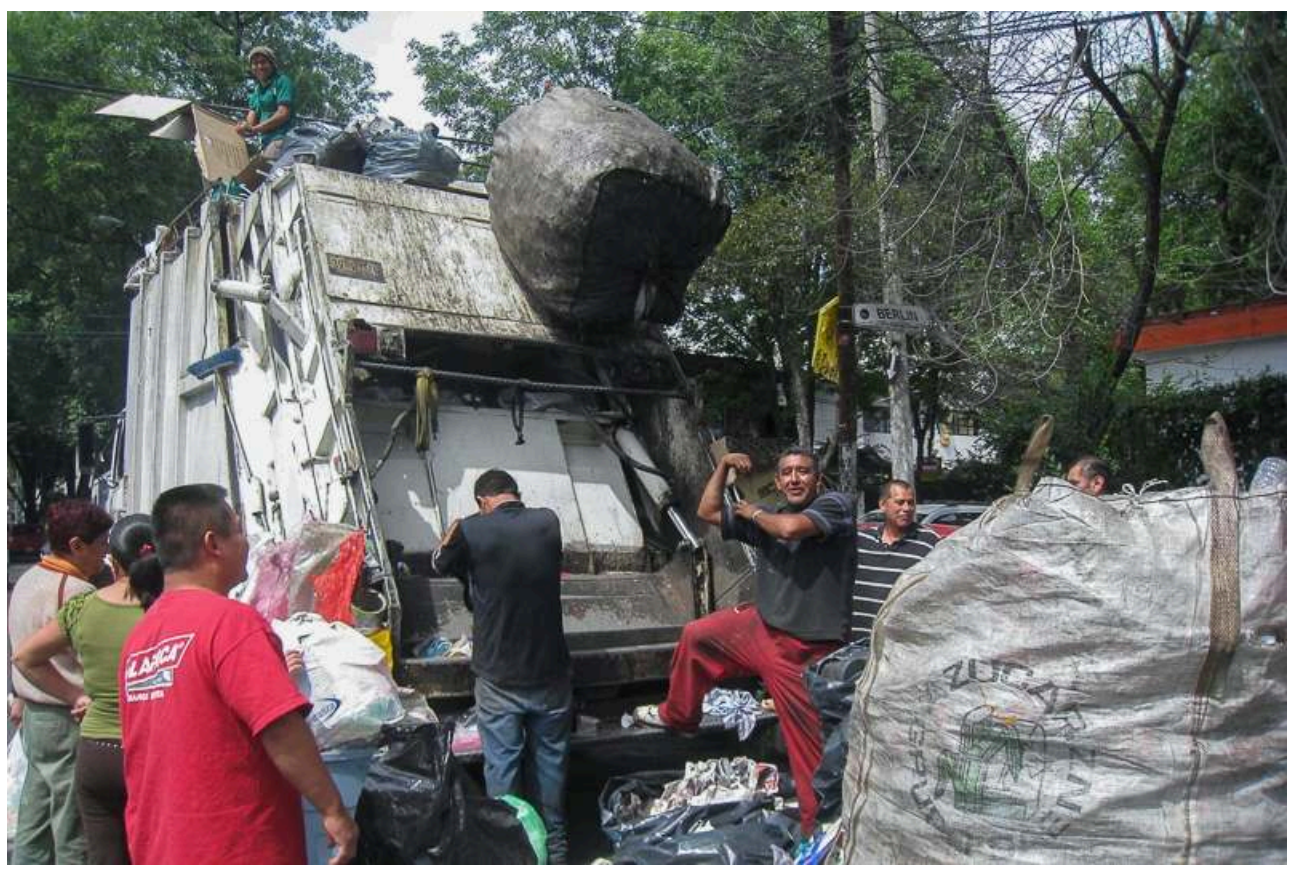

Le travailleur au centre de l'image est l'un des 10000 « volontaires » qui réalisent la collecte et la récupération des déchets dans la capitale mexicaine. Ceux-ci gagnent leur vie grâce aux pourboires des riverains et à la vente des matériaux récupérés. À Mexico, le système de ramassage est pris en charge à la fois par les services de propreté de la ville et par des récupérateurs appelés pepenadores. En réponse à ma demande de le photographier, cet homme prend la pose en montrant ses muscles, exposant la virilité de son travail. Sa posture évoque la force : force pour soulever les poids, force pour résister aux dangers associés à la manipulation de matières souillées indissociables de ce métier. L'énorme sac noir rempli de canettes au-dessus de sa tête évoque bien, symboliquement, les risques du métier.

Mexico D.F., Mexique, mars 2012. Auteur : C. Cirelli. 


\section{Illustration 3 - Dona Graça e o seu carrinh/ Dona Graça et son chariot}

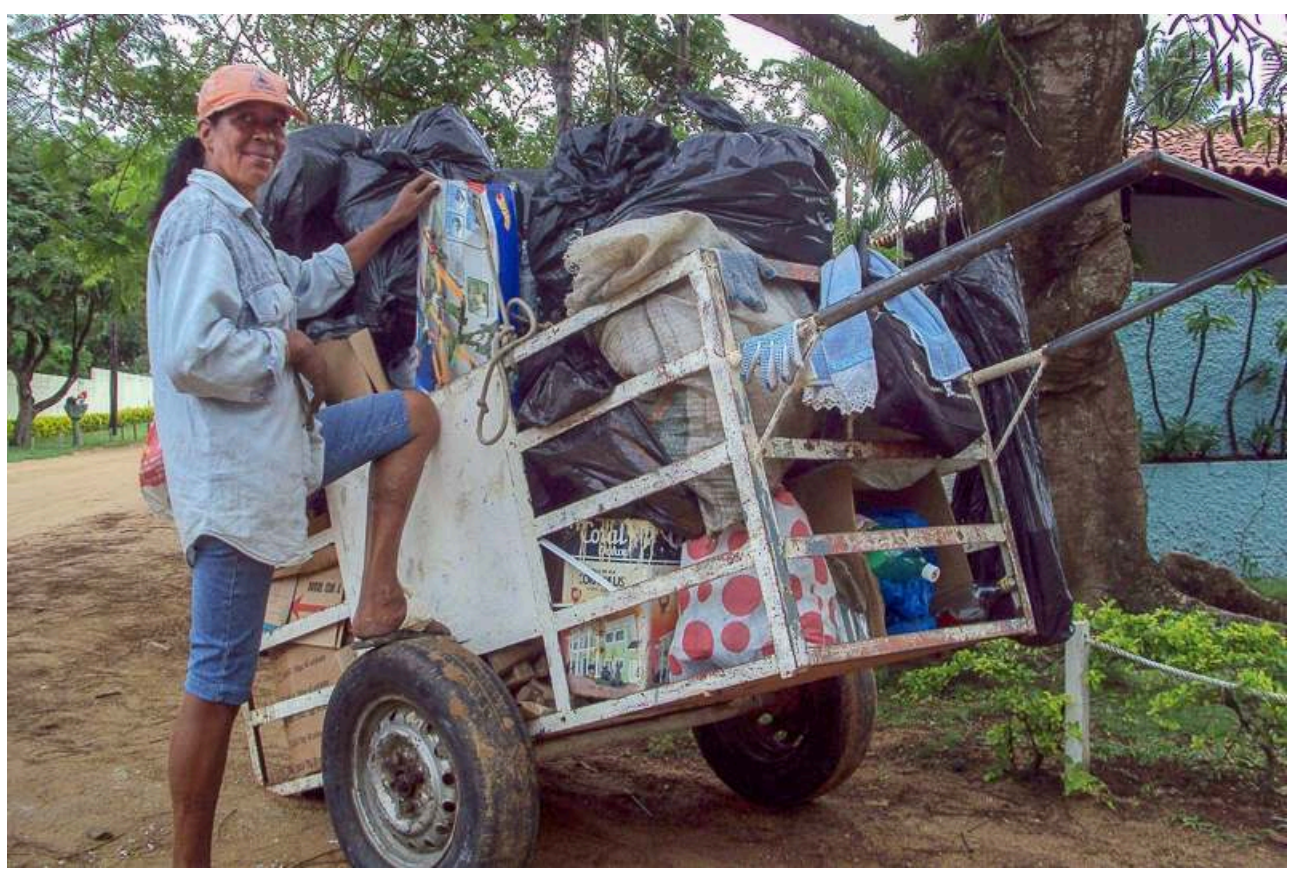

Dona Graça, qui n'a jamais travaillé dans le secteur formel, se présente comme une recicladora. Elle est très fière de son chariot blanc qui est le symbole de son indépendance. Comme dona Graça pratique la récupération dans ce quartier depuis plusieurs années maintenant, elle explique que « des gens séparent exprès les trucs pour [elle] ». Ces récupérations-là se révèlent les plus fructueuses bien que, paradoxalement, dona Graça soit également contrainte d'embarquer des choses qui l'intéressent moins. Mais qu'importe : le fait que les habitants aient préalablement séparé leurs déchets et prévu un sac spécifique pour les emballages induit une inestimable amélioration de son travail de récupération C'est beaucoup plus rapide et, surtout, infiniment moins dégradant car les matériaux sont propres. On n'a plus le sentiment d'agir à la dérobée, mais l'impression tangible d'être engagé dans une interaction respectueuse.

Vitória, Espírito Santo, Brésil, juillet 2010. Auteur : J. Cavé 
Illustration 4 - La dignité à travers les poubelles

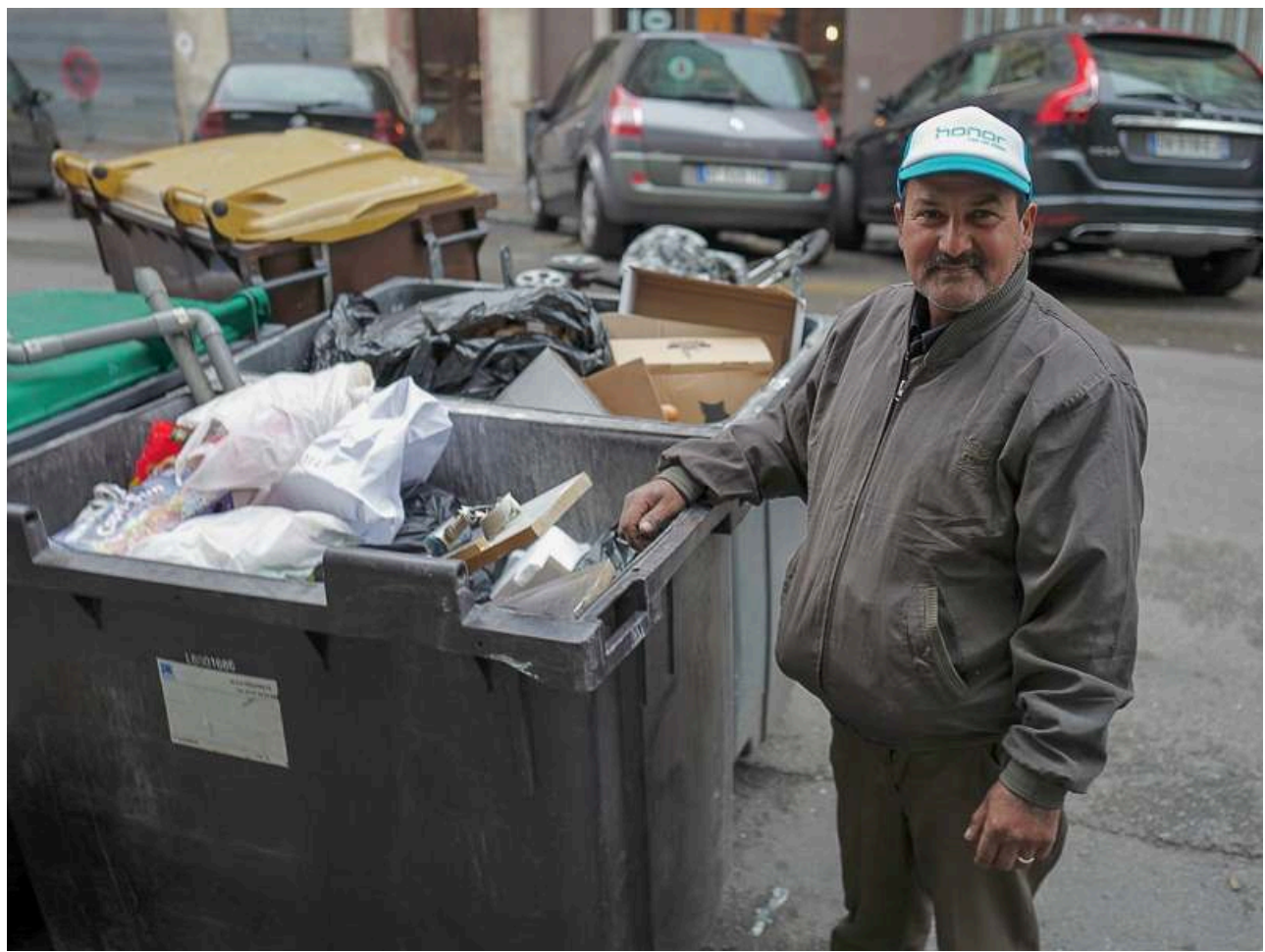

J'ai rencontré cet homme à Marseille par une froide matinée de février. Rom de Roumanie, il est venu en France pour fuir la pauvreté de son pays et il vit aujourd'hui avec sa famille dans un squat du quartier du Panier. Ne trouvant pas de travail, il gagne sa vie en cherchant dans les bacs à ordures des vieux fils électriques dont il extrait le cuivre pour le revendre au poids. C'est lui qui a voulu poser ainsi et, en prenant cette photo, j'ai eu l'impression de réconcilier l'inconciliable : montrer la dignité d'un homme qui fouille dans les poubelles. ${ }^{4}$

Marseille, France, février 2016. Auteur : P. Garret. 


\section{Illustration 5 - Le parcours de Yunus}

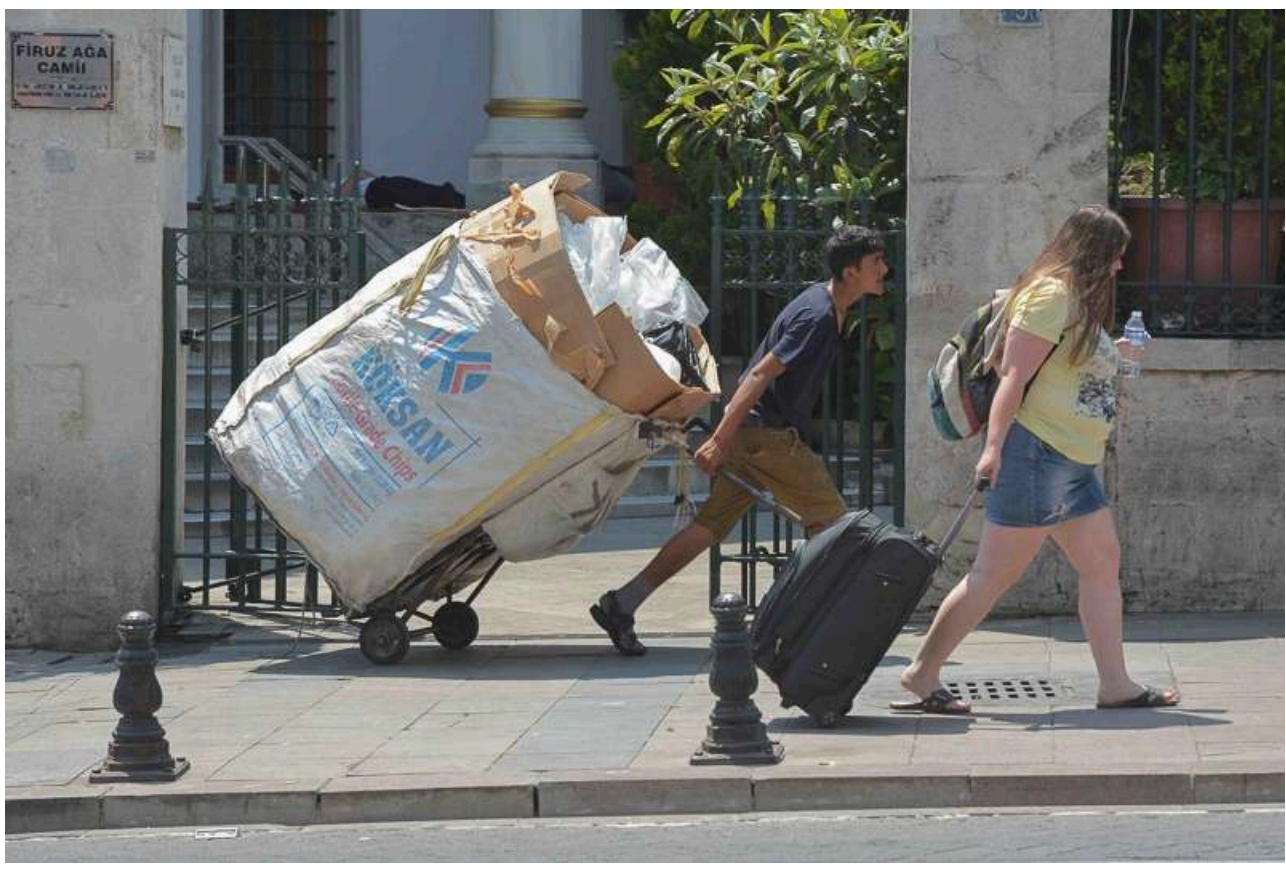

Yunus, jeune récupérateur turc, est en train de revenir de l'une de ses tournées quotidiennes de récupération dans les rues d'Istanbul, comme ici dans le quartier hyper touristique de Sultan Ahmet. La photographie met en exergue le contraste saisissant entre deux mondes parallèles : alors que Yunus, courbé vers l'avant, tire d'un pas rapide le lourd fardeau issu de sa collecte, la touriste devant lui traîne tranquillement sa valise, marchant en claquettes d'une allure flegmatique. Chacune des tournées de Yunus dure environ deux heures pour près de dix kilomètres parcourus et il en effectue en moyenne quatre à cinq dans la journée, six jours par semaine. Agé d'environ 16 ans, il fait partie d'un groupe de jeunes récupérateurs qui se sont installés dans une friche urbaine du quartier de Süleymaniye. Cette dizaine de jeunes hommes, tous originaires de la région d'Aksaray (environ 700 km d'Istanbul), a acheté un camion en commun et s'organise sous la forme d'une coopérative, sans patron.

Istanbul, Turquie, juillet 2015. Auteur : P. Garret/MuCEM, terrain réalisé avec B. Florin. 
Illustration 6 - Lunch

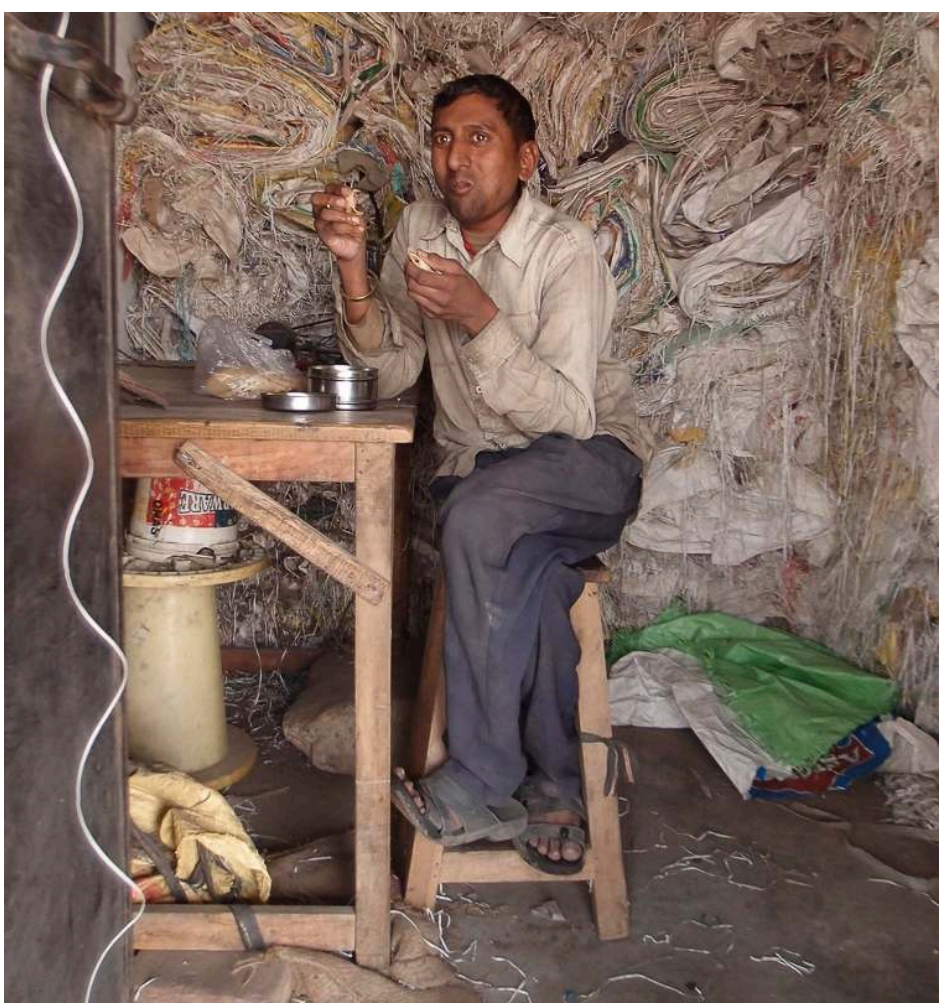

Cet homme déjeune dans sa petite boutique à Nangloi, un quartier populaire à l'ouest de Delhi, implantée juste en face d'un bidonville de waste pickers (chiffonniers). Son travail consiste à récupérer et réparer des grands sacs en plastique, roulés et empilés derrière lui, qu'il revend ensuite aux habitants du bidonville. Ce sont ces larges et épais sacs que les waste pickers portent sur leur dos et qu'ils remplissent de déchets glanés informellement lors de leurs pérégrinations en ville, parvenant à récupérer en fin de journée d'importants volumes de matériaux recyclables. L'une des revendications des organisations de protection de waste pickers vise à la reconnaissance par les autorités de leur contribution au nettoiement de l'espace public, notamment par la fourniture d'un équipement plus adéquat. En l'absence d'intégration de ces travailleurs, ces simples sacs s'imposent comme l'un des outils essentiels de la collecte des déchets et, de manière symbolique, incarnent ici la précarité du travail de la récupération.

Nangloi, Delhi, Inde, janvier 2016. Auteur : R. de Bercegol. 


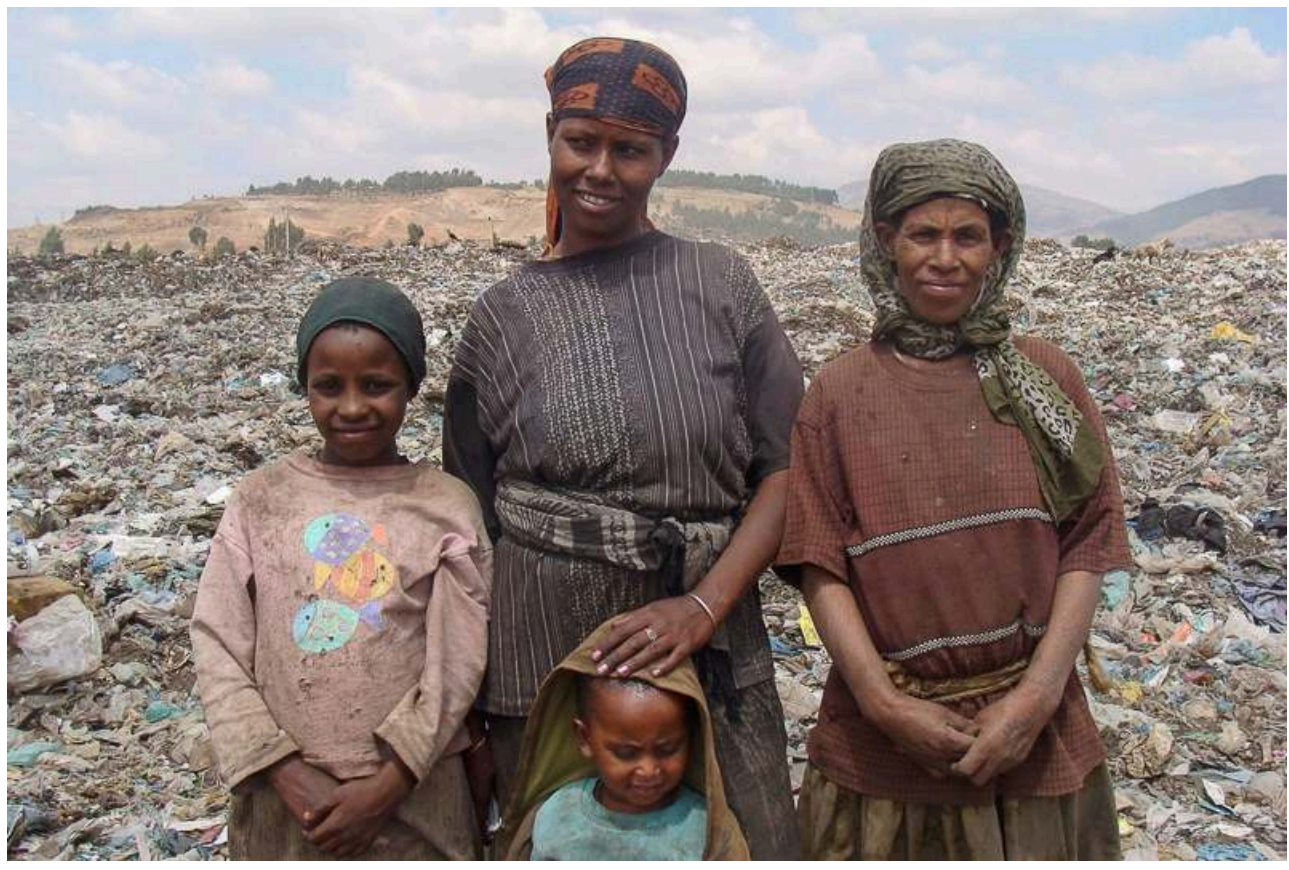

Ces « travailleurs des déchets » sont les membres d'une même famille, on les appelle Kosheman, ce qui signifie en amharique " ceux qui travaillent avec les koshasha », c'est-à-dire les ordures. Ils sont la face cachée de la capitale éthiopienne qui connaît des transformations sans précédent, portée par le slogan Clean and green Addis Ababa. Cette photographie est le résultat d'un long processus d'intégration au sein de ces travailleurs, méfiants et peu désireux d'interagir avec moi. Après plusieurs semaines de présence sur le site, j'ai pu, peu à peu, sortir mes notes, les interroger, les photographier... à leur demande. Tant et si bien qu'ils se sont parfois confiés. Cette photographie évoque avant tout la solidarité, non seulement entre les plus démunis, mais aussi entre les générations qui se côtoient sur la décharge.

Addis Abeba, Éthiopie, mai 2009. Auteur : A. Pierrat.

\section{Savoir-faire : le tri, le démontage, le nettoyage}

11 Les portraits ci-dessous montrent ces hommes et femmes en activité dans leurs différents lieux de travail qu'il s'agisse des quartiers périphériques, des dépôts des grossistes plus centraux, occupant des dents creuses ou des délaissés urbains, ou encore des entrepôts utilisés par les coopératives. Dans ces lieux d'arrivée de la collecte, les matériaux sont triés, notamment lorsque leur qualité est disparate, classés, puis pesés par catégorie afin de pouvoir payer le récupérateur au plus juste du prix fixé par le grossiste lorsqu'il travaille pour son propre compte. Dans le cas des coopératives, qui cherchent à ajouter de la valeur au travail de récupération en développant d'autres phases de la valorisation des déchets, la pesée est réalisée pour estimer les quantités de matériaux récupérés qui entrent à l'entrepôt et qui sont ensuite transformés, avant leur vente. Quel que soit le cas, le récupérateur connaît bien la valeur de son chargement et, de son côté, l'acheteur sait évaluer au mieux de ses intérêts la qualité, la quantité et, bien sûr aussi, la valeur des matériaux apportés.

12 Échappant définitivement à leur statut de déchets, ces ressources sont triées par types, par couleurs et sont prêtes à être démontées, désossées, nettoyées, lavées, compactées, pour être transformées sur place ou revendues aux ateliers de recyclage. Les travailleurs présentés jouent un rôle indispensable dans le fonctionnement du circuit 
de la récupération puisqu'ils se situent à la charnière entre, d'une part, le travail des récupérateurs réalisé en dehors du système de gestion des déchets dit officiel et, d'autre part, l'économie dite formelle, grâce à la transformation d'un matériau - le déchet récupéré - en un intrant industriel (Suárez et Schamber, 2007). Les techniques et outils diffèrent d'un lieu à l'autre mais, partout, les savoir-faire sont manifestes : la dextérité, la rapidité, la connaissance des divers composants participent au processus de valorisation. Ceci n'empêche pas la pénibilité de certaines tâches répétitives, les positions inconfortables, le poids des charges à manipuler ainsi que les revenus très irréguliers, parfois très faibles (notamment en ce qui concerne les plus jeunes) selon les situations.

D'un point de vue de l'organisation du travail, dans certains contextes, en particulier en Amérique Latine, les autorités publiques ont accepté d'encadrer la pratique de récupération puis de l'intégrer dans les programmes de gestion des ordures grâce à des contrats pour le ramassage public ou la récupération des matières recyclables. Adhérer à une coopérative a permis à ces travailleurs de laisser derrière eux des conditions de travail et de vie très dures, d'être incorporés au processus productif avec des droits sociaux, des conditions de travail plus dignes et plus sûres, parfois un subside à un salaire minimal. De même, ils ont augmenté leurs revenus grâce à la valeur plus importante des matières récupérées, alors traitées et assemblées par les coopératives au lieu d'être vendues à des intermédiaires à très bas prix. L'adhésion aux coopératives a signifié non seulement l'accès à des droits mais également, dans certains contextes, la participation des récupérateurs aux débats sur la politique des déchets. Toutefois, si les exemples ci-dessus témoignent qu'une partie des récupérateurs a pu faire évoluer leurs conditions de travail, pour beaucoup d'autres persistent des situations de profonde fragilité en raison de conditions de travail (et de vie) bien plus précaires. Ces travailleurs n'ont pas de sécurité sociale ou d'emploi; ils se heurtent à de nombreux obstacles pour s'organiser librement et sont soumis à des relations fortement hiérarchiques et clientélistes.

Cette indéniable inégalité des positions de ces « travailleurs des déchets », la nature des échanges entre ces derniers et les chercheurs au moment du terrain transparaissent dans les portraits réalisés dans cette série du portfolio: gestes, sourires, anecdotes, blagues, fières poses... 
Illustration 8 - Sous le pont

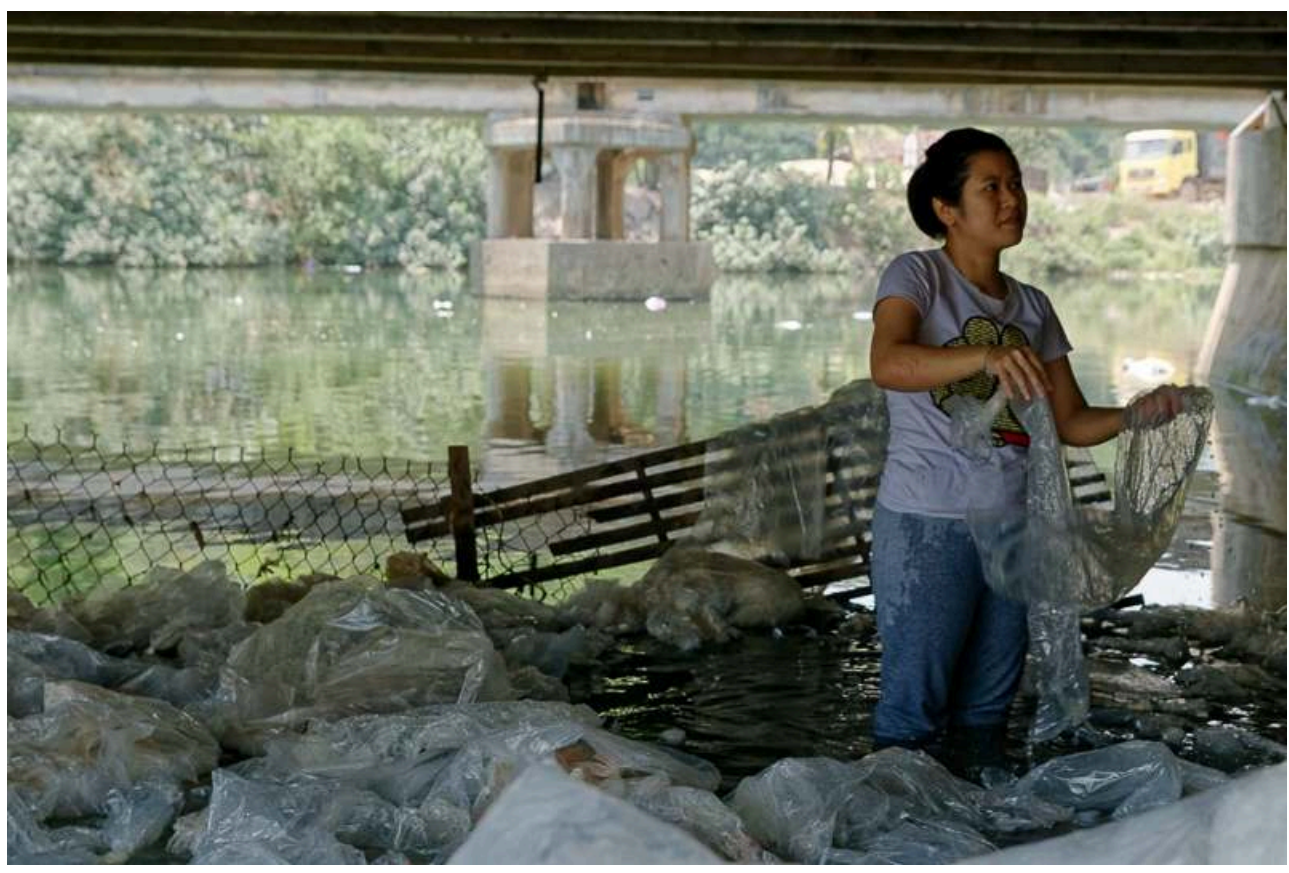

Après plusieurs enquêtes sur la question des déchets et la pollution plastique au Vietnam, j'ai pris I'habitude de regarder sous les ponts, car on y trouve souvent des amas d'ordures. Un jour, mon regard a été attiré par des plastiques qui flottaient sous une route très fréquentée des environs de Quy Nhon, une ville côtière du centre du pays. En descendant sous le pont, je n'imaginais pas rencontrer une mère et sa fille, dans l'eau jusqu'aux genoux, le dos courbé, occupées à rincer des sacs transparents dans l'eau de la rivière. Afin de compléter les revenus agricoles de la famille, elles récupèrent au port des sacs ayant contenu des fruits de mer, les lavent dans la rivière pour les débarrasser de leur odeur et les revendent à l'une des usines de recyclage de plastique qui ont récemment ouvert dans la région.

Quy Nhon, Binh Dinh, Vietnam, mars 2016. Auteur : M. Le Meur. 
Illustration 9 - La jeune fille à la perle

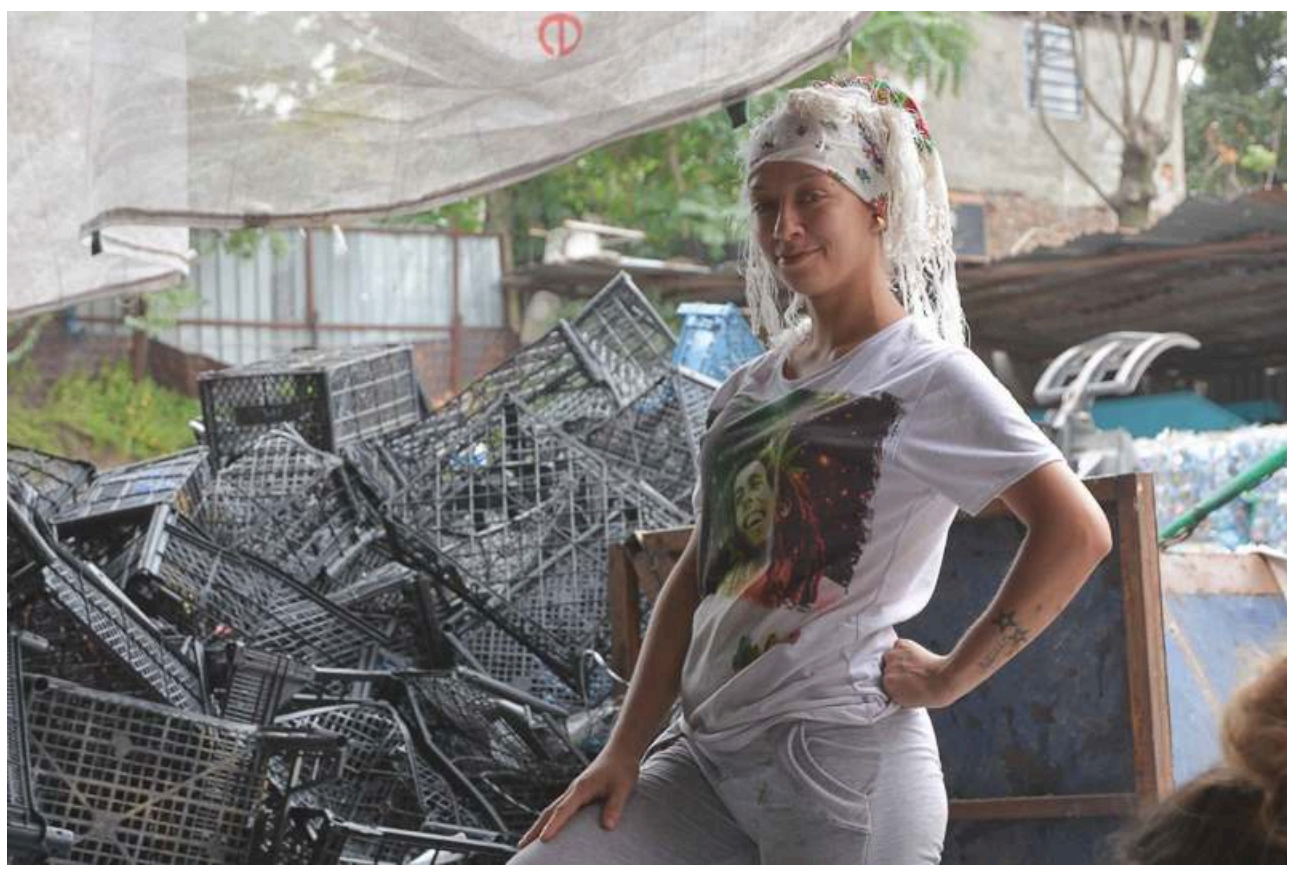

À Istanbul, peu de femmes travaillent à la récupération des déchets dans les rues. Ici, cette jeune femme rom de Turquie trie les déchets en plastique avec plusieurs autres femmes de sa communauté dans un des grands dépôts du quartier Süleymaniye. Elle nous a expliqué qu'elle préférait l'activité de tri à celle de la récupération, plus sale, et ce dans l'espace protecteur du dépôt plutôt que dans la rue. Malgré des conditions de travail difficiles, nous avons été très bien accueillis par ces femmes qui, après un long entretien, ont toutes pris plaisir à poser pour le photographe avec une fierté non dissimulée. Cette jeune femme a choisi elle-même cette pose, posture qui nous évoque a posteriori celle de « La jeune fille à la perle », célèbre tableau de J. Vermeer (1665).

Istanbul, Turquie, juillet 2015. Auteur : P. Garret, terrain réalisé avec B. Florin. 
Illustration 10 - Vers la reconnaissance des « travailleurs des déchets »

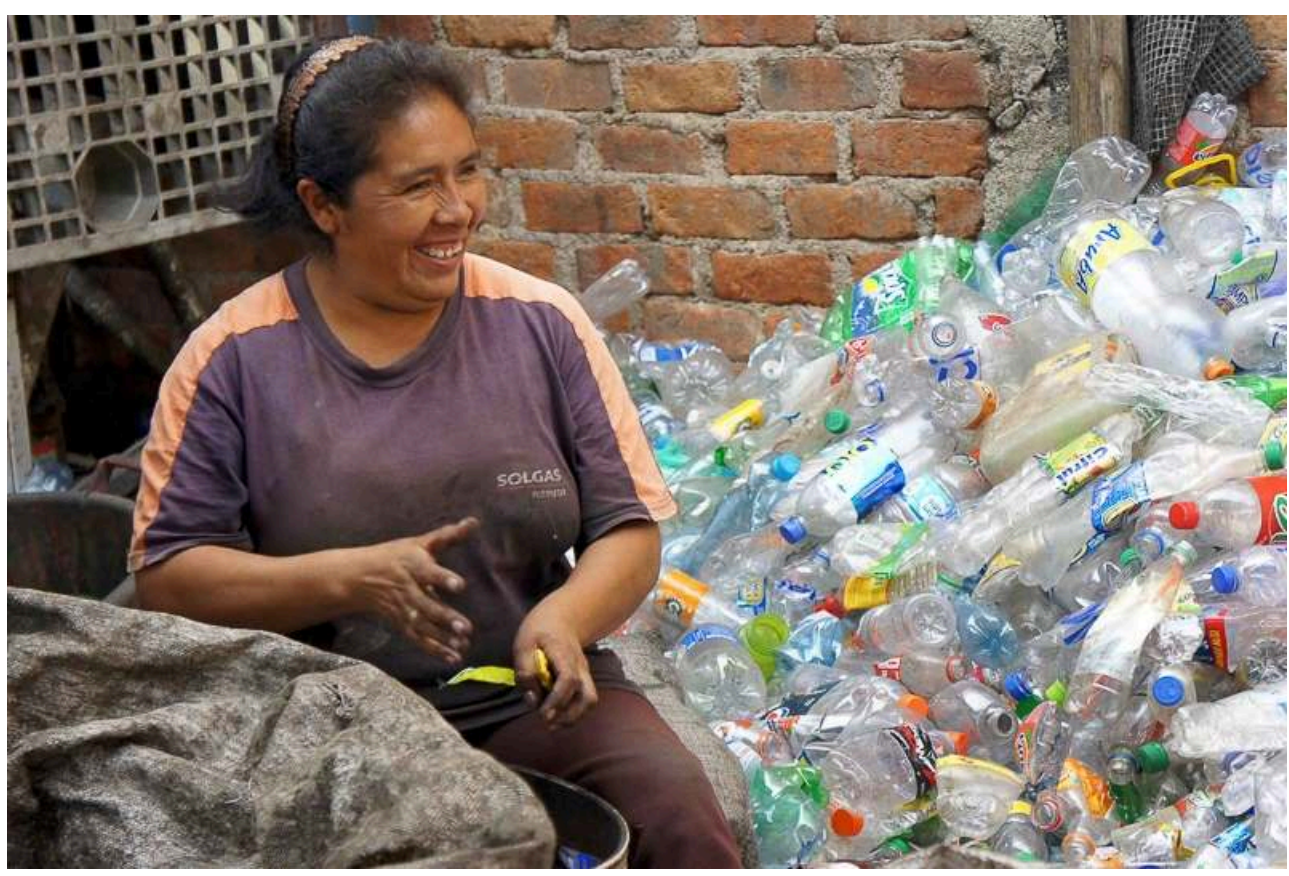

Les récupérateurs informels collectent les matériaux recyclables en ouvrant les sacs-poubelles dans les rues, alors que ceux qui sont formels, travaillant en collaboration avec la municipalité et les ONG, récupèrent les sacs de déchets recyclables directement auprès des ménages. Cette distinction entre informels et formels découle de la « Loi du Recycleur » d'octobre 2009 qui offre un cadre pour leur formalisation. Forts de cette expérience, certains récupérateurs vont jusqu'à créer leur microentreprise d'achat de déchets recyclables qui seront pré-transformés en vue de leur exportation vers la Chine ou les États-Unis. Cette photo a été prise lors d'une visite, organisée par l'ONG péruvienne Alternativa, dans l'une de ces micro-entreprises de pré-transformation des déchets. Le sourire de la travailleuse affectée au nettoyage des bouteilles illustre ici la progressive reconnaissance du travail des déchets et l'espoir pour la formalisation du secteur.

Lima, Pérou, mars 2014. Auteur : M. Rateau. 
Illustration 11 - Le jeune laveur de bouteilles

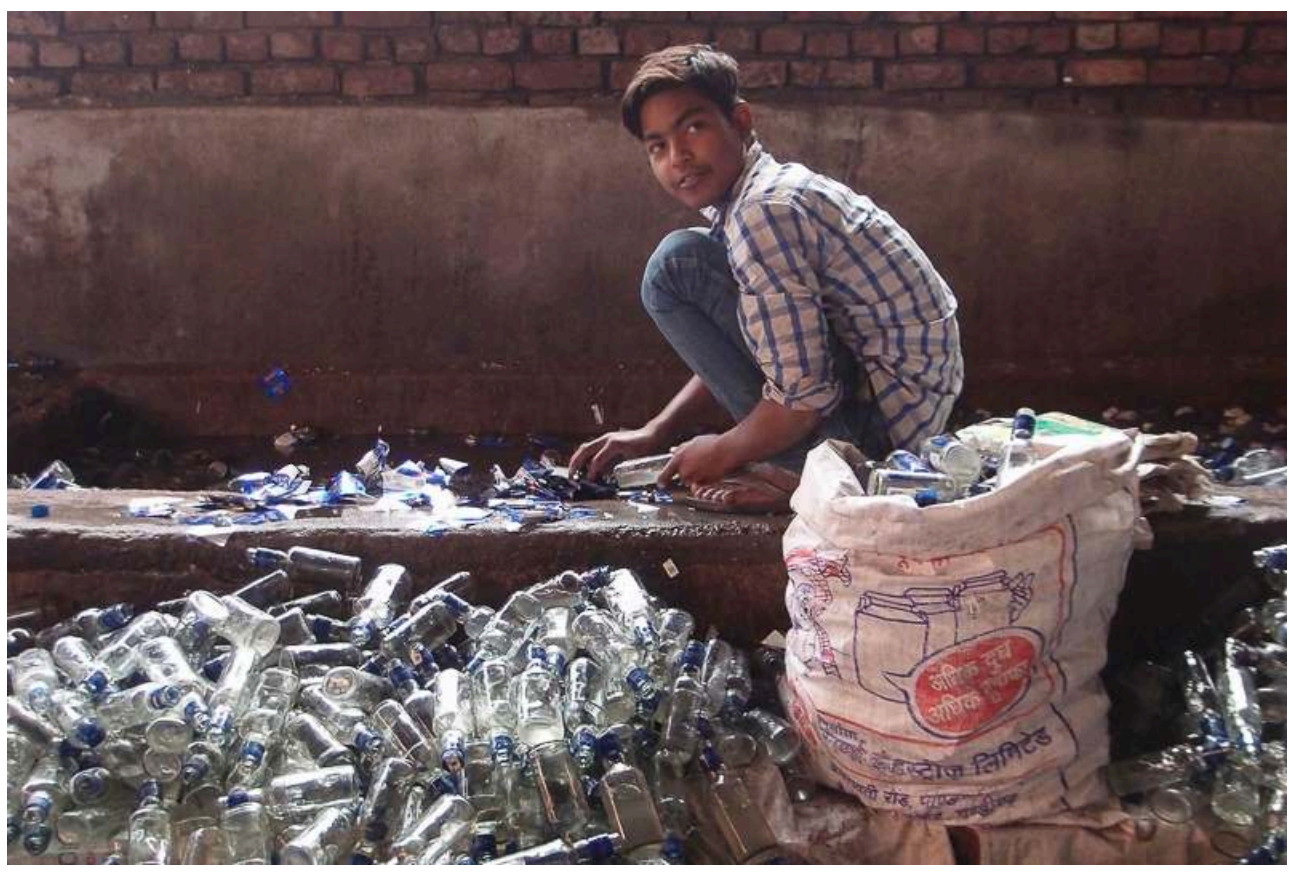

Mohan dit qu'il a quinze ans, mais il n'en est pas tout à fait certain. II vient d'un petit village de l'Uttar Pradesh, un État pauvre d'Inde du Nord. II a trouvé un emploi à Kanchan Kunj, un quartier au sud de Delhi, dans un hangar de récupération de bouteilles de verre. Les bouteilles usagées y sont amenées par camion, triées selon la marque et la couleur du verre, lavées à la main puis, finalement, revendues à une usine d'embouteillement. Mohan travaille aux étapes de lavage : il plonge une par une les bouteilles dans un bain d'eau savonneuse, les nettoie rapidement et en retire l'étiquette. Il est payé au nombre de bouteilles et ne s'est pas arrêté de frotter alors que nous discutions. Lorsque je reviendrai quelques jours plus tard à l'usine pour lui donner son portrait imprimé, je le trouverai dans la même position, accroupi au-dessus de son bac, et il s'essuiera à peine les mains pour courir montrer sa photographie à son grand-frère.

Delhi, Inde, janvier 2016. Auteur : R. de Bercegol. 
Illustration 12 - La maison-benne du vieux couturier

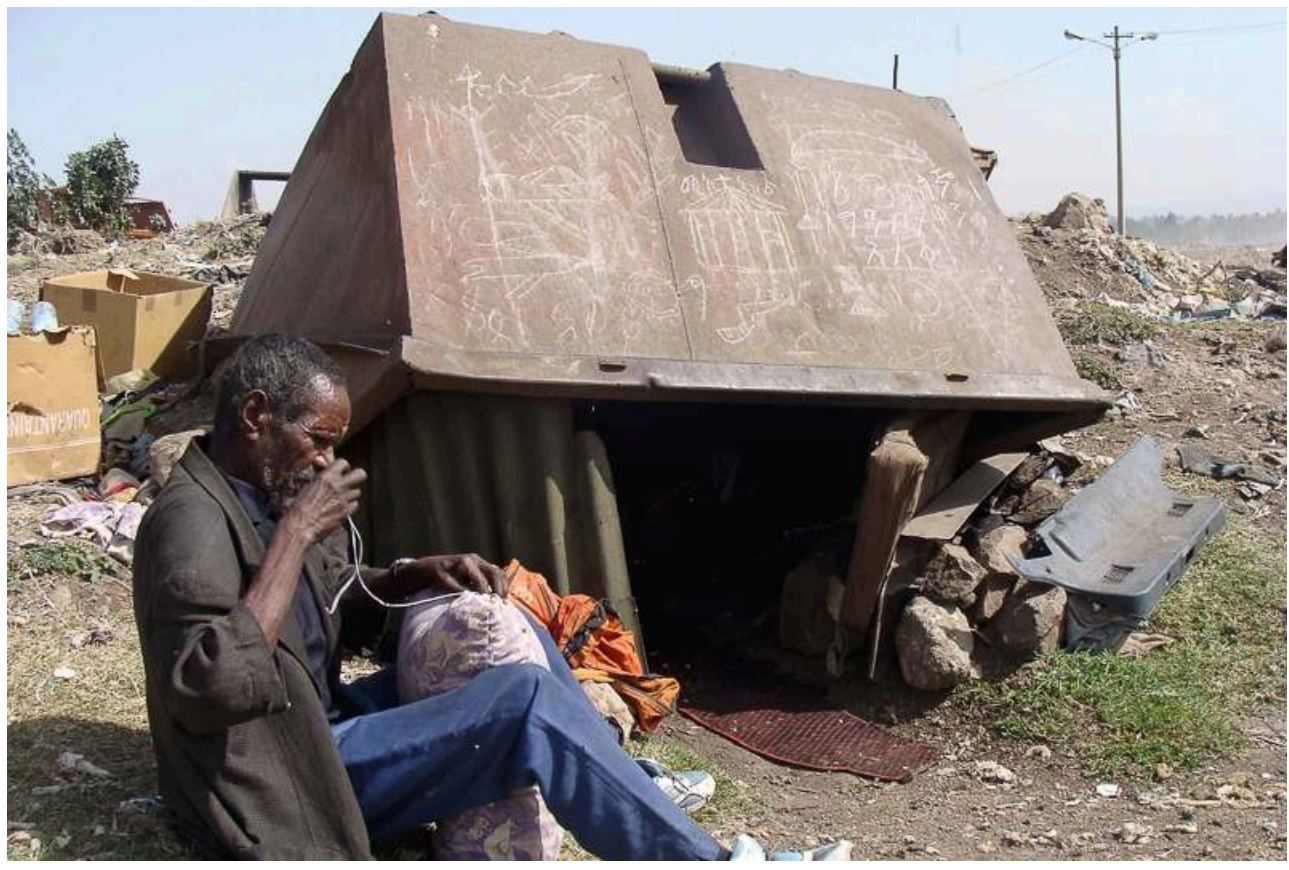

Tesfaye Belaynen, 53 ans, est fabricant d'oreillers à Koshe Repi depuis 24 ans : Avant j'habitais près de Dässé dans le Wällo (centre-nord du pays). Je suis arrivé à Addis Abeba lors de la famine de 1983-1985. Avant j'étais soldat. Je n'ai pas trouvé de travail, alors j'habite ici, regarde à l'intérieur... Oui, la färänj (l'étrangère) elle peut regarder, je fais de l'artisanat. À partir des bouts de tissus qu'il récupère, stocke et achète parfois aux récupérateurs, il fabrique en moyenne deux oreillers par jour. On aperçoit, au second plan, une benne retournée « aménagée » qui est l'habitation du vieil homme. En me permettant de photographier son refuge, ce récupérateur nous donne à voir son intimité et sa précarité.

Addis Abeba, Éthiopie, avril 2009. Auteur : A. Pierrat. 
Illustration 13 - De l'objet aux matériaux, démontage d'écrans CRT à Nyayo Market

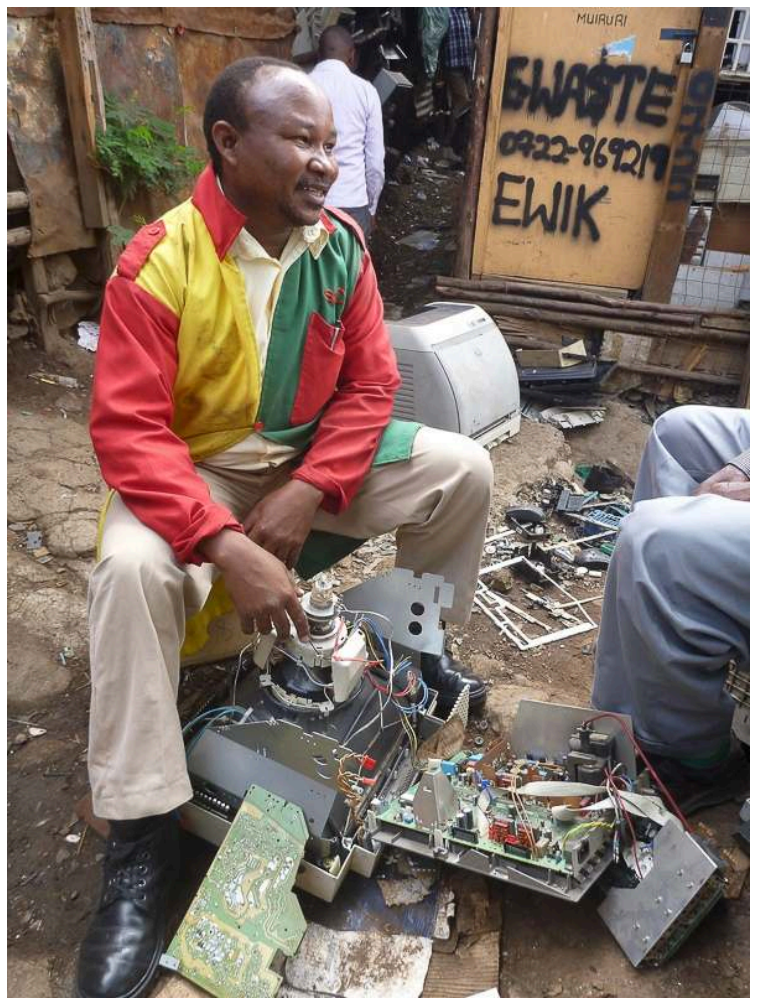

À Nairobi, le marché de Nyayo Market est l'un des points névralgiques de la filière de revalorisation des appareils électriques et électroniques. Originaire de l'ouest du Kenya, Gideon y occupe un atelier spécialisé dans le matériel informatique. Il a longtemps gagné sa vie en revendant localement et en exportant vers l'Ouganda des écrans à tubes cathodiques (CRT) de seconde main. Mais ils sont aujourd'hui démodés et Gideon préfère les démonter : leur valeur se trouve en effet dans le cuivre et les métaux précieux contenus dans les cartes électroniques qui les composent et qui partiront pour le recyclage vers I'Asie ou l'Europe. Derrière lui, l'atelier d'un autre fundi (technicien en swahili) qui préside E-Waste Initiative Kenya (EWIK), une association dont l'objectif est d'organiser et faire reconnaître leur travail.

Nairobi, Kenya, février 2017. Auteur : R. Reboux. 


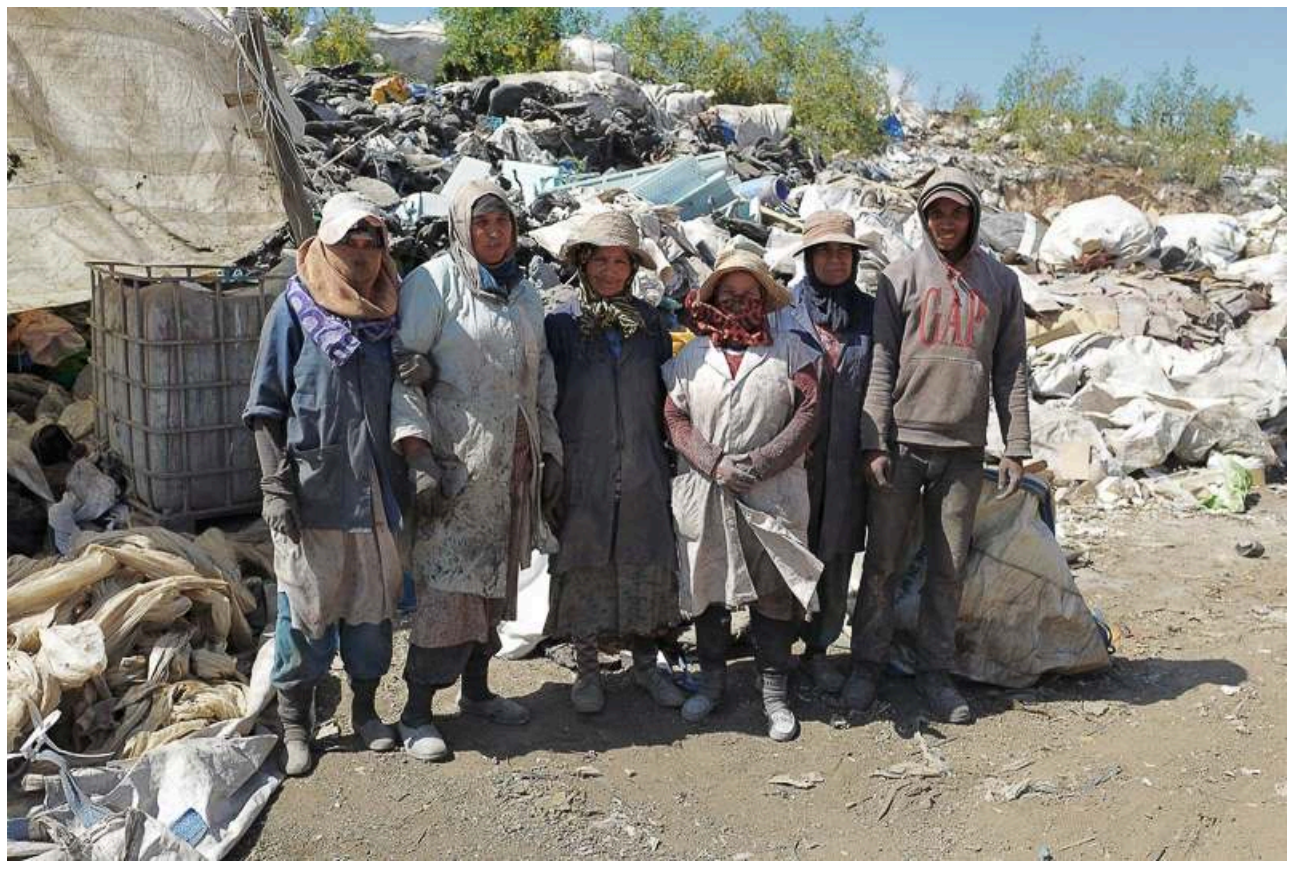

À Casablanca, les femmes ne collectent pas dans les rues et elles sont employées exclusivement au tri des matériaux ramenés par les hommes. Souvent originaires de la campagne, elles y font des allers-retours en fonction des récoltes. Elles gagnent peu mais leurs revenus constituent un appoint à l'économie familiale et leur permettent de survivre lorsqu'elles sont seules. Les entretiens et prises de vue avec ces femmes et cet homme ont été faciles à réaliser et riches d'informations : il y a de leur part une très forte demande de reconnaissance de leur place dans la société urbaine, de leur rôle dans l'économie du recyclage et en faveur de l'environnement.

Casablanca, Maroc, mai 2016. Auteur : P. Garret, terrain réalisé avec B. Florin.

\section{Le recyclage, la commercialisation et les circulations}

15 Ajouter de la valeur aux activités de récupération par des opérations de transformation et traitement des matières récupérées est l'un des défis majeurs auxquels sont confrontés les récupérateurs afin d'augmenter " la chaîne de valeur du recyclage » (Scheinberg et Simpson 2015 ; Jaligot et al., 2016). Les récupérateurs sont souvent en début de la chaîne en collectant les matériaux sales et les vendant à des prix relativement faibles ainsi que nous l'avons évoqué précédemment et auxquels d'autres acteurs ajoutent de la valeur. Pouvoir investir dans des équipements, même rudimentaires, avoir accès à l'eau, à l'électricité ou à la propriété des terrains où sont établis les entrepôts ou les espaces de travail, représentent des conditions fondamentales pour améliorer et faire évoluer l'activité de valorisation des déchets et leurs performances.

16 Les ateliers comprennent les activités de transformation des matériaux en produits semi-finis ou finis avant leur commercialisation. Ils supposent un investissement en machines-outils ainsi qu'une spécialisation professionnelle accrue. Chez les chiffonniers du Caire, le nombre d'ateliers s'élève à près d'un millier et les matériels de recyclage - parfois imités d'un prototype acheté à l'étranger!-, y sont souvent fabriqués à partir de matériaux eux-mêmes issus de la récupération. La fabrication d'objets en plastique prêts à l'utilisation (bassines, seaux, cintres, etc.) s'est fortement 
développée depuis deux décennies. Au Caire, comme dans les zones industrielles de Delhi ou encore dans les "villages de métiers " d'Hanoi spécialisés dans le recyclage (Ird-images, 2018), on trouve toutes les activités de traitement, compression en balles, empaquetage des produits semi-finis (papiers, cartons, métaux, etc.) destinés aux usines du secteur formel.

Dans d'autres situations, la transformation des matériaux récupérés est réalisée dans les mêmes lieux où l'on trie et l'on nettoie, comme c'est le cas pour certaines des coopératives argentines qui - afin d'ajouter de la valeur à la chaîne du recyclage réalisent également le broyage du plastique et la production de granulés vendus directement aux entreprises nationales.

Les revenus des récupérateurs et recycleurs résultent ainsi de différentes sources : la vente directe des matériaux récupérés, la vente des matériaux transformés (en matière première secondaire ou en produits) et, dans certains contextes, notamment en Amérique Latine, où les récupérateurs ont su gagner des espaces politiques et générer un cadre de légalité pour développer leur travail, des subsides octroyés par les pouvoirs publics. Au début des années 2000, par exemple, à Buenos Aires, les autorités locales ont déclaré le recyclage et l'activité de récupération légaux : quatorze coopératives se sont alors constituées.

Dans tous les cas étudiés, ce processus confirme les liens entre les activités du secteur informel et celles du secteur formel: le premier alimente le second. Il en découle un continuum (Cirelli et Florin 2015; Jaglin et al., 2018; Scheimberg et al., 2011; Scheimberg et al., 2016) qui se matérialise par la circulation des matériaux, de l'argent et des acteurs. Cependant, cette inclusion de la force et du produit du travail des hommes et des femmes du secteur informel ne s'accompagne que trop rarement de droits sociaux, de droits du travail ou de revenus plus équitables. La dernière série de ce portfolio donne à voir cette étape de la valorisation des matériaux. Elle donne aussi à voir celles et ceux qui revendiquent davantage de reconnaissance pour leur contribution à l'économie des déchets. 
Illustration 15 - El trabajo no es basura / Notre travail n'est pas sale

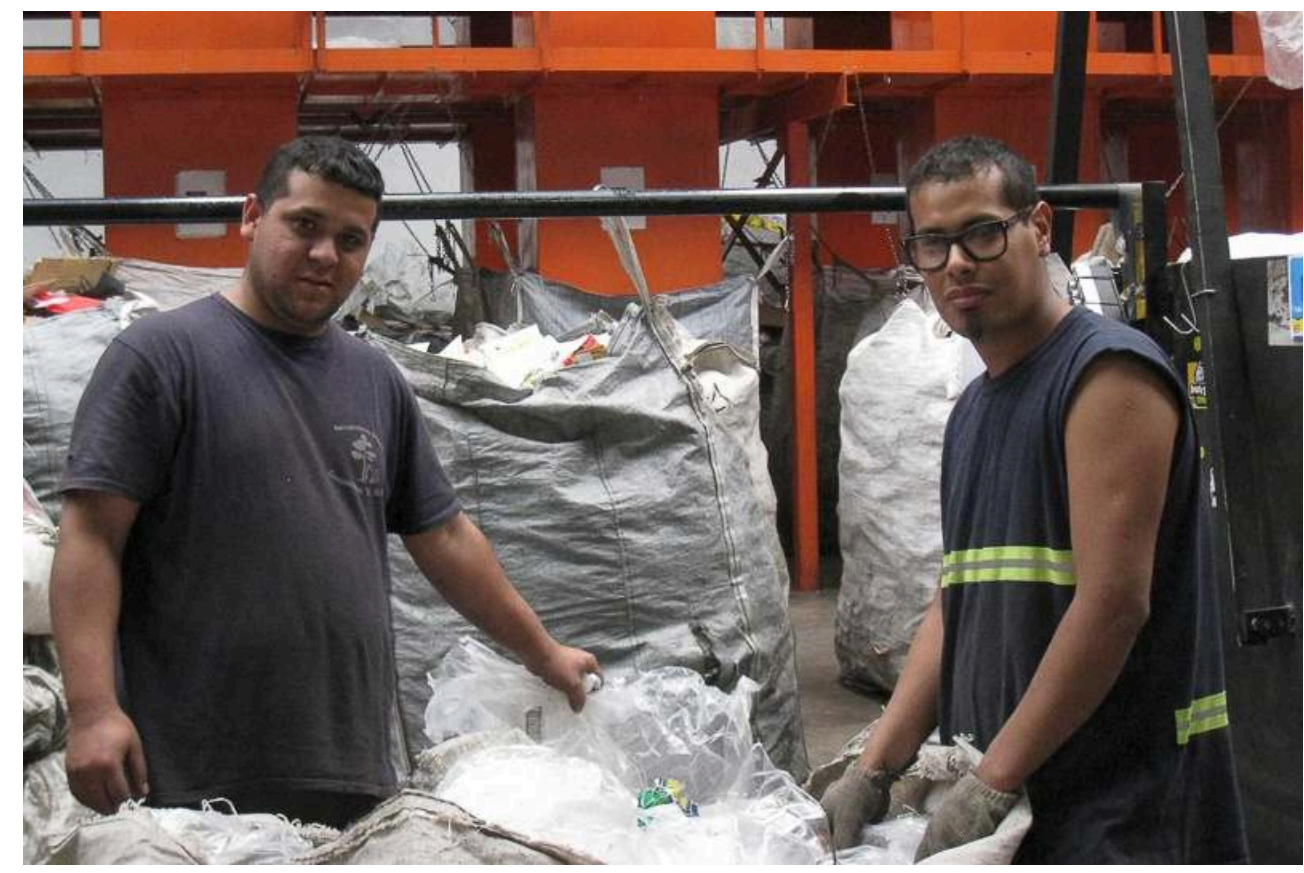

Ce cliché est pris dans les locaux de la coopérative El Álamo, l'une des douze coopératives intégrées actuellement au service public de propreté urbaine de la ville de Buenos Aires. Ces deux membres de la coopérative sont en train de trier du plastique et, comme leurs autres collègues, considèrent que le travail avec les rebuts «n'est pas sale » mais recèle une valeur. En effet, la coopérative traite 200 tonnes de déchets par mois. Son travail, associé à celui des autres associations de recuperadores, représente des économies importantes pour la municipalité qui, normalement, paie par tonne de résidus enfouis. En Argentine, au cours des dernières années, ces coopératives ont fait des progrès significatifs : leur pari a été non seulement d'améliorer les conditions de travail de leurs membres, les capacités de collecte, recyclage et commercialisation des déchets (en court-circuitant les intermédiaires), mais également de gagner une plus grande visibilité pour que leur contribution à l'économie urbaine et à l'environnement soit reconnue.

Buenos Aires, Argentine, novembre 2015. Auteur : C. Cirelli. 
Illustration 16 - Pak Warjito, dit « le chef des biffins »

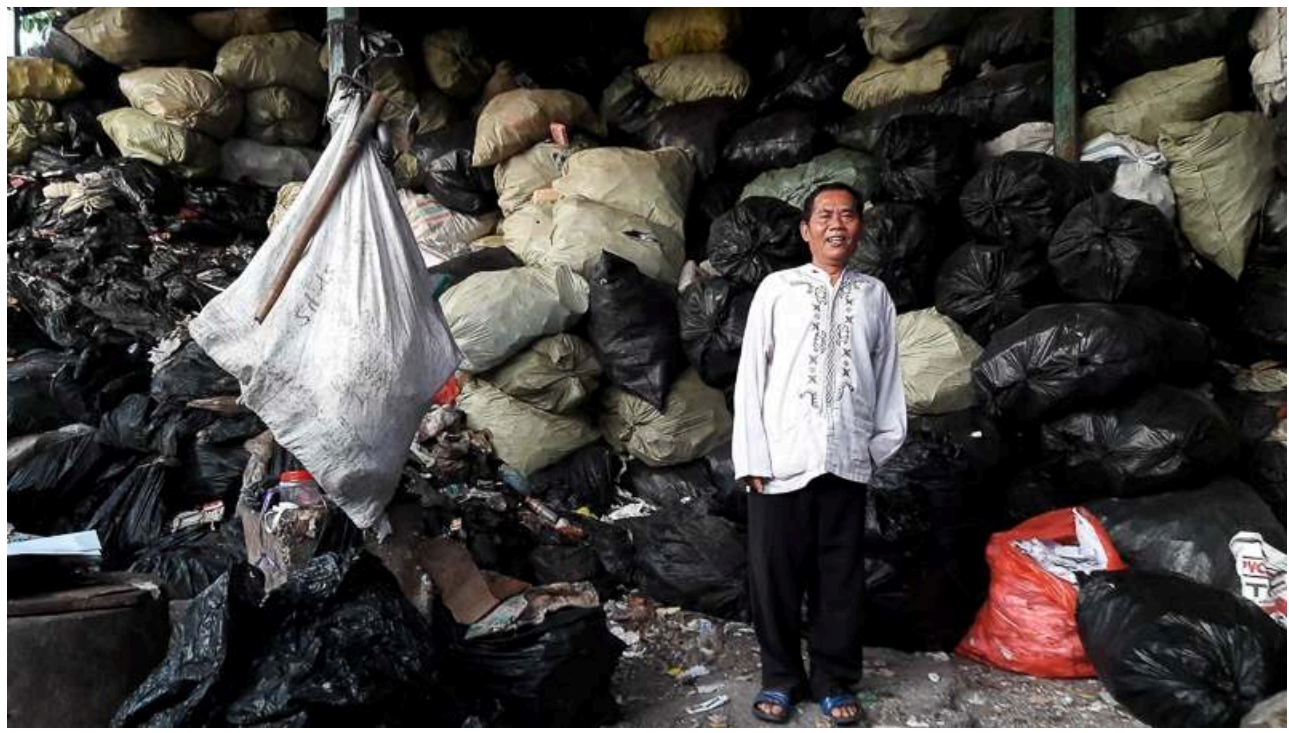

Pak Warjito est un négociant, surnommé « le Chef des biffins ». Avant, il tirait un chariot, mais désormais à la tête d'une équipe de 20 personnes, il collecte les déchets de centres commerciaux, d'hôpitaux, de zoos et il est payé pour cela : il prend 40 \% des revenus et ses équipes récupèrent les 60 \% restants, plus les déchets. Son équipe brasse $150 \mathrm{~m}^{3}$ de déchets par jour grâce à cinq camions et quatre pick-ups. Ils apportent chaque jour 4 à 7 tonnes de déchets résiduels au centre d'enfouissement. Pour le reste, il vend l'équivalent de 800000 roupies indonésiennes par jour (moins de 50 euros) à des usines de l'agglomération : principalement des bouteilles en verre et des papiersplastiques. Depuis ses débuts, Pak Warjito s'est considérablement enrichi : il s'est construit une belle maison et a fait cinq fois le pèlerinage à la Mecque ! Ses travailleurs, eux, restent logés dans des cabanes sur pilotis au-dessus des monceaux de sacs poubelles parcourus par d'énormes rats...

Surabaya, Java, avril 2016. Auteur : J. Cavé. 
Illustration 17 - L'atelier de recyclage de 'Atef

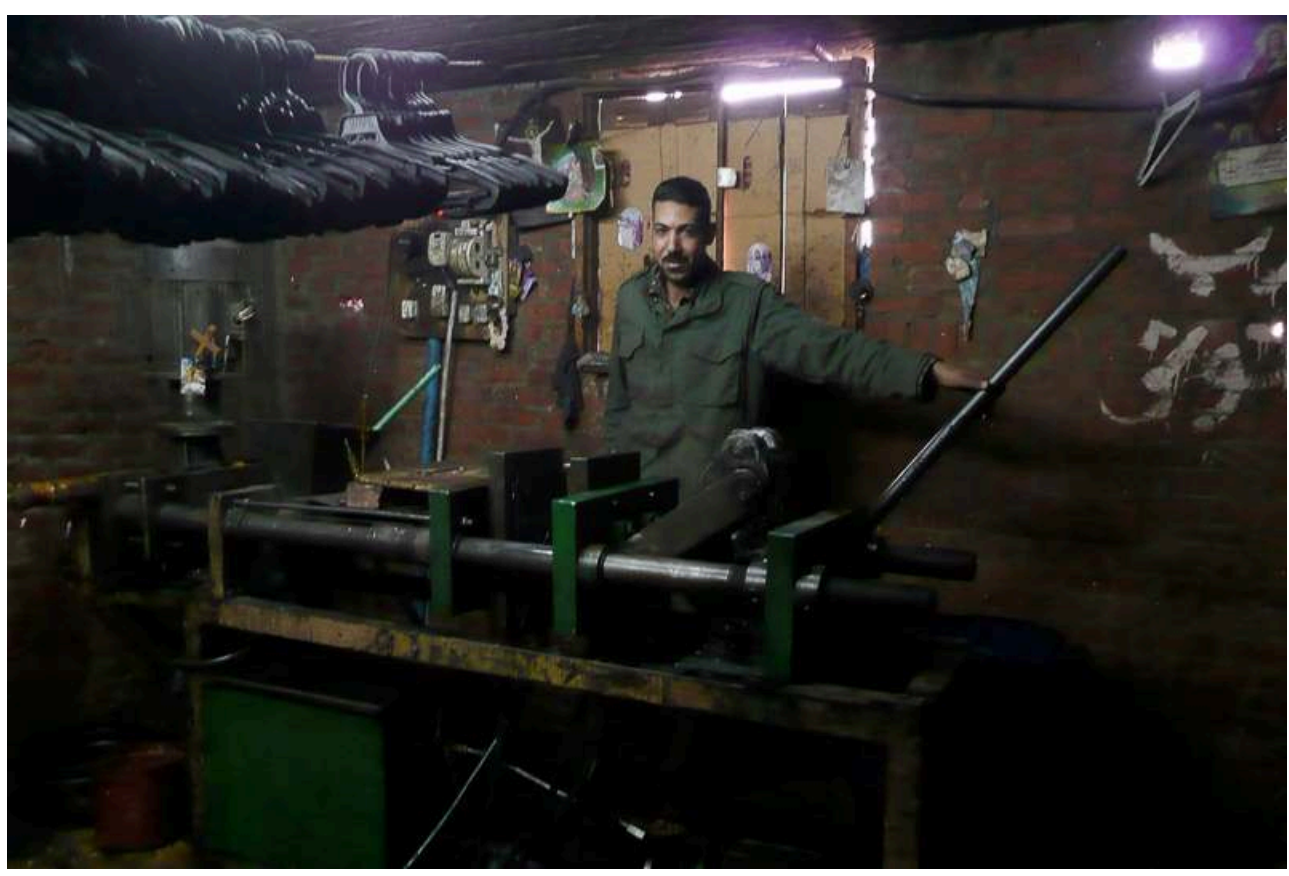

Au Caire, à Manchiat Nasser, 'Atef récupère ou achète du plastique noir à ses voisins chiffonniers pour le recycler en cintres vendus à des grossistes du centre-ville. Après avoir longtemps travaillé comme porteur de sacs, il devient indépendant en 2004 en achetant pour 800 euros une machine à mouler les porte-manteaux. Depuis, il parvient à gagner environ 80 euros par mois. Si le chiffonnier du Caire représente pour beaucoup une figure archaïque de la pauvreté, le parcours de 'Atef témoigne que les positions sociales et économiques sont très diverses et que sa communauté est loin d'être homogène. Les savoir-faire, initiatives et inventions sont de mise dans les activités des chiffonniers: loin d'être les «éboueurs » de la ville, ils sont avant tout des recycleurs et des commerçants. Ce portrait veut ainsi témoigner de cet aspect de leur métier.

Le Caire, Égypte, février 2008. Auteur : B. Florin. 
Illustration 18 - Le millefeuille du ferrailleur

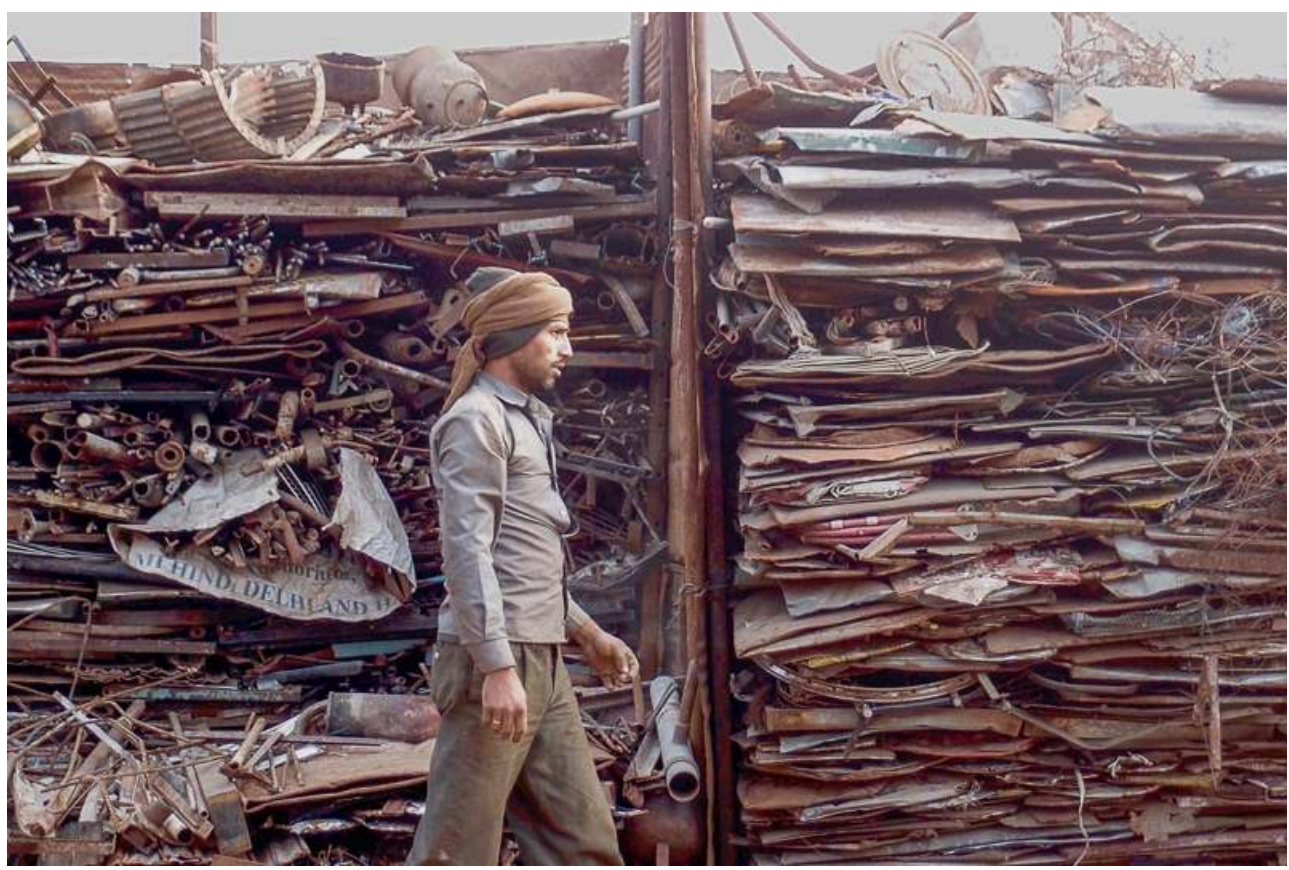

Nasim passe devant un amas de tôles froissées et empilées selon une typologie bien précise. La

ferraille est classée en fonction de sa composition (fer, fonte, acier) et séparée des autres métaux non ferreux (comme le cuivre, l'inox, le zinc, le bronze, l'aluminium). Pendant notre passage, Nasim prépare un chargement de fer qui alimentera les fonderies de Muzaffarnagar, une ville du nord de l'Inde à une centaine de kilomètres de Delhi. Avec d'autres ouvriers, il porte à mains nues de lourds panneaux de fer à l'arrière du camion. L'épaisse peau de ses mains, coupées à de multiples endroits, porte le stigmate de son travail éreintant.

Delhi, Inde, février 2016. Auteur : R. de Bercegol. 
Illustration 19 - La fonderie d'aluminium de Barakat

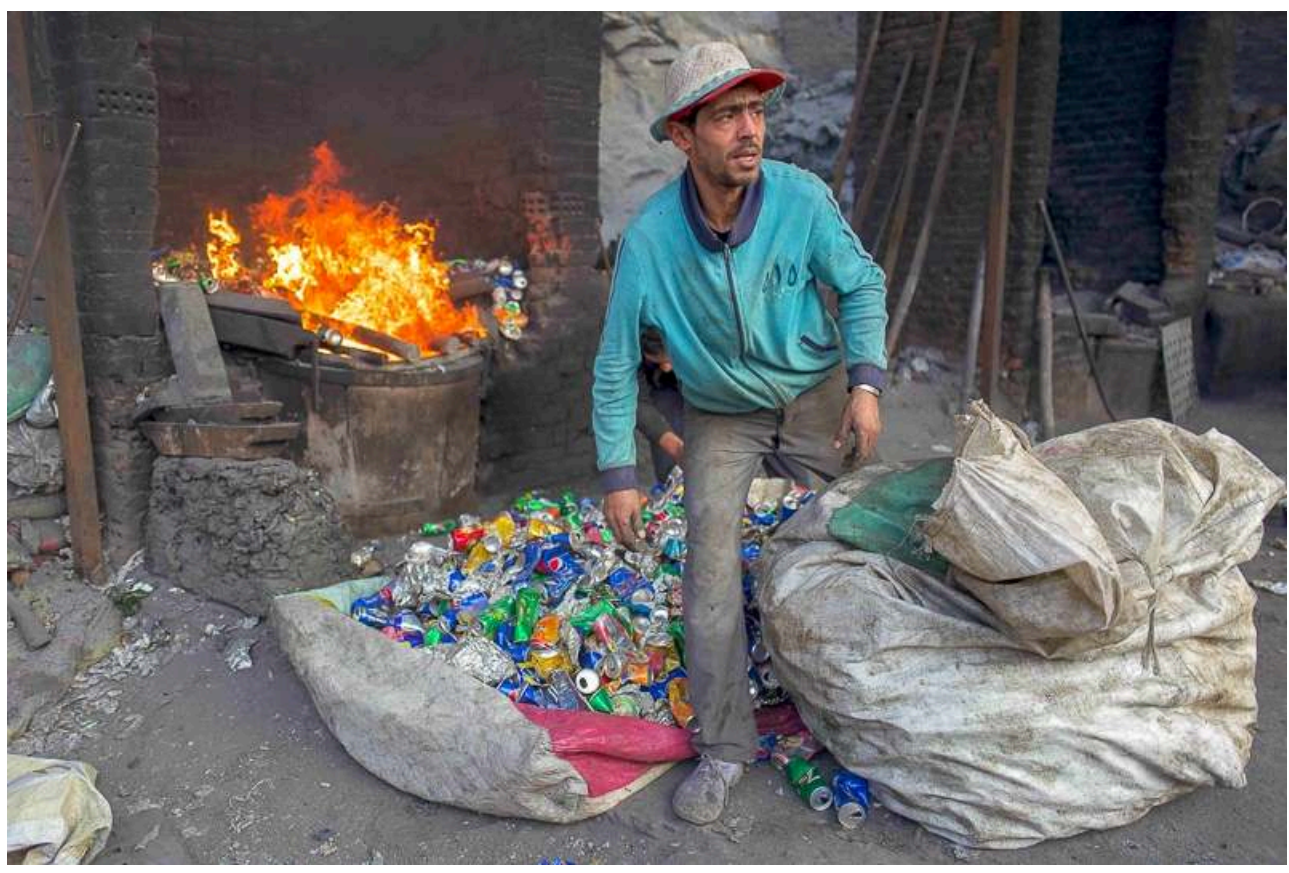

Les zabbâlîn égyptiens (le mot dérive de zabbâl, le déchet) ne se contentent pas de collecter et trier les déchets, ils transforment eux-mêmes certaines des matières récoltées comme l'aluminium. Le système de fonte de l'aluminium est très simple : un creuset en ciment réfractaire avec un système de chauffe dont le carburant est de l'huile de vidange. Une fois le creuset mis en chauffe, les ouvriers vont y déverser les canettes : la chaleur est intense, comme le montre cette photo. Une fois liquéfié, l'aluminium sera coulé dans des moules en forme de lingots qui seront revendus à bon prix. Le Caire, Égypte, février 2017. Auteur : P. Garret, terrain réalisé avec B. Florin. 
Illustration 20 - À la scierie de pare-chocs

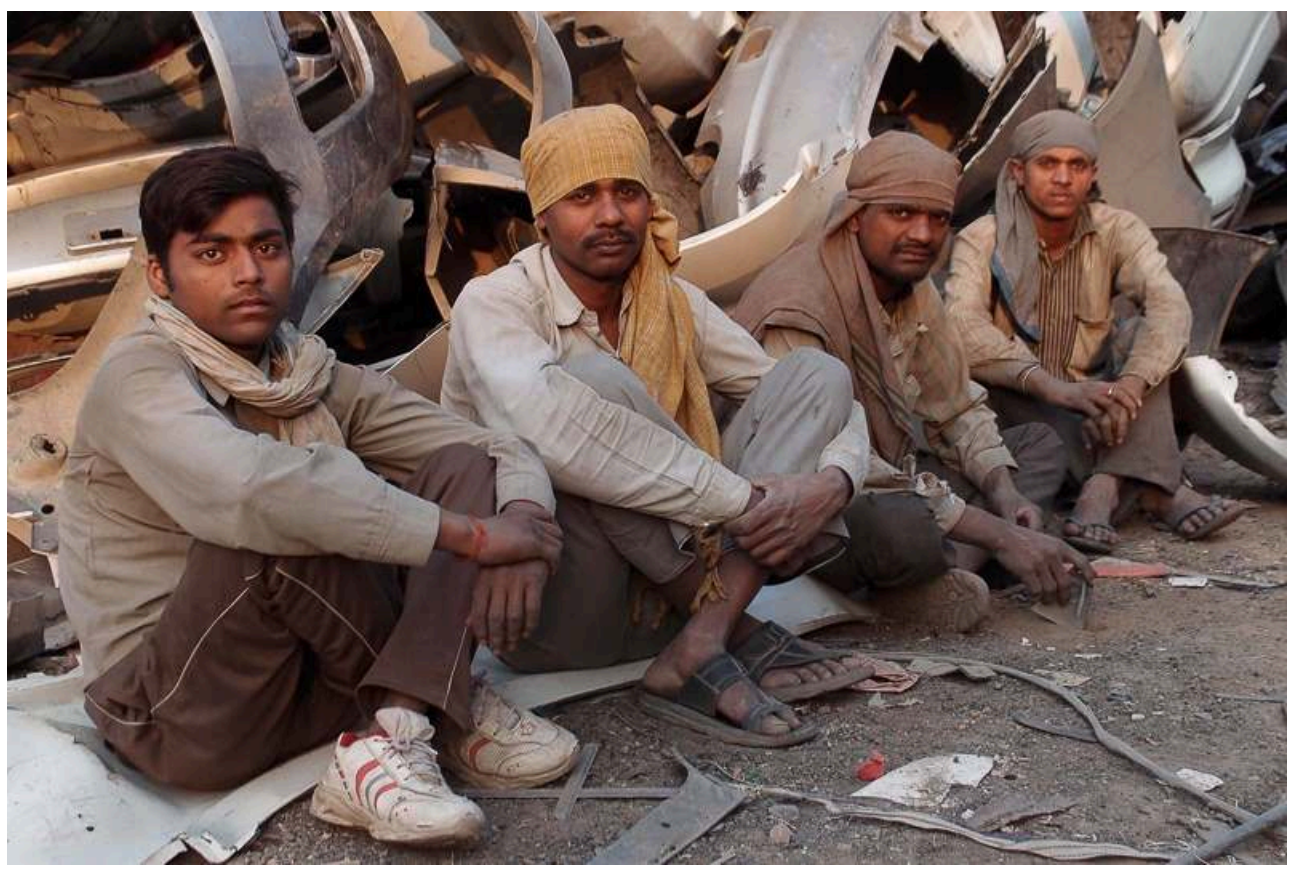

Ces quatre ouvriers sont employés dans une scierie de pare-chocs de voitures, à Tikri Kalan, au sein du «PVC market », un marché spécifiquement dédié à la revente de plastiques en gros, où sont acheminées chaque jour des tonnes de matériaux qui y sont triés, affinés et revendus. Leur travail consiste à découper et à broyer les pare-chocs que l'on aperçoit derrière eux. II s'agit d'une activité très physique, particulièrement pénible et dangereuse. Leur contremaître les a autorisés à prendre une pause rapide afin que nous puissions discuter quelques instants.

Comme une majorité des travailleurs du "PVC Market", ils sont issus de la même caste Khatik, une communauté traditionnellement associée au travail impur de la tannerie et reléguée au bas de la société hindoue. Depuis plusieurs décennies, ce groupe a néanmoins su développer un nouveau savoir-faire, capable de différencier tous types de plastiques (polypropylène, polyéthylène, polyvinyl chloride, polyuréthane, etc.) et certains Khatik ont ainsi pu fortement s'enrichir grâce à la valorisation de cette connaissance (Gill, 2012). Ces quatre hommes ne gagnent en revanche qu'un bien maigre salaire, de l'ordre de 10000 roupies (équivalent à moins de 200 euros par mois), mais n'oseront pas s'en plaindre devant leur employeur. Ce dernier sonne déjà la fin du repos et, très vite, c'est le retour au brouhaha de la scierie.

Delhi, Inde, janvier 2016. Auteur : R. de Bercegol 


\section{Illustration 21 - Mustapha, patron d'une gelssa}

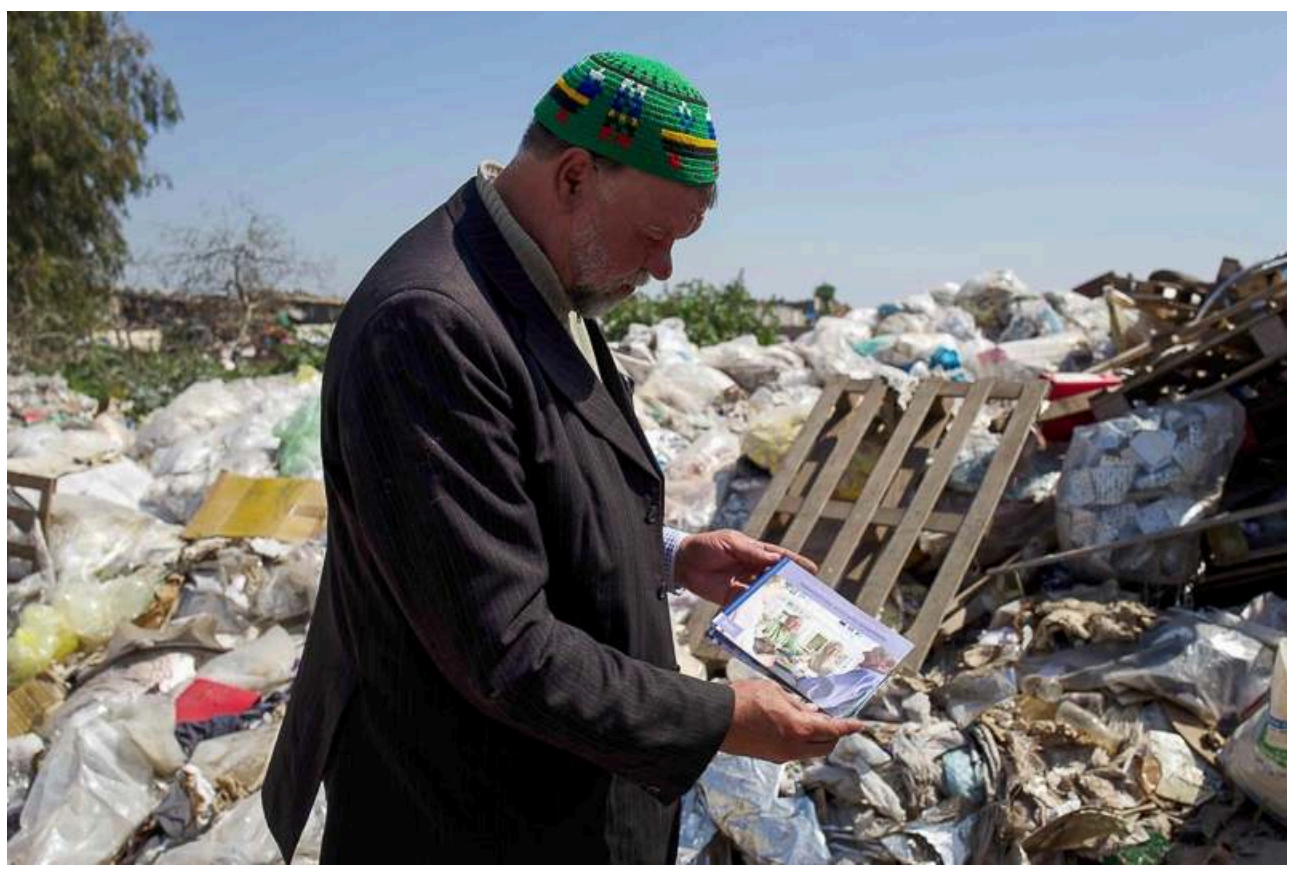

Mustapha est le patron d'une gelssa (terme dérivé du verbe gels, « s'asseoir » en dialecte marocain) du quartier de Lahraouine à Casablanca. Les gelssas sont des enclos de tailles diverses entourés de palissades où sont triés et transformés les matériaux ramenés par les récupérateurs de rue. Mustapha a tenté de créer une "Association de défense des commerçants des matériaux recyclés » afin de plaider leur reconnaissance auprès des pouvoirs publics : Le but c'était d'aider les gens et d'encadrer les activités en s'organisant en association pour accéder à des droits et aux soins. C'est un métier pas reconnu, mais les gens sont vraiment motivés pour se défendre (...). Si l'association n'a pas été validée par les autorités, Mustapha continue tant bien que mal à militer : On contribue à l'économie du pays, c'est grâce à nous que c'est recyclé, sinon, ça serait brûlé.

Casablanca, Maroc, avril 2017. Auteur : P. Garret, terrain réalisé avec B. Florin. 
Illustration 22 - Moolchand

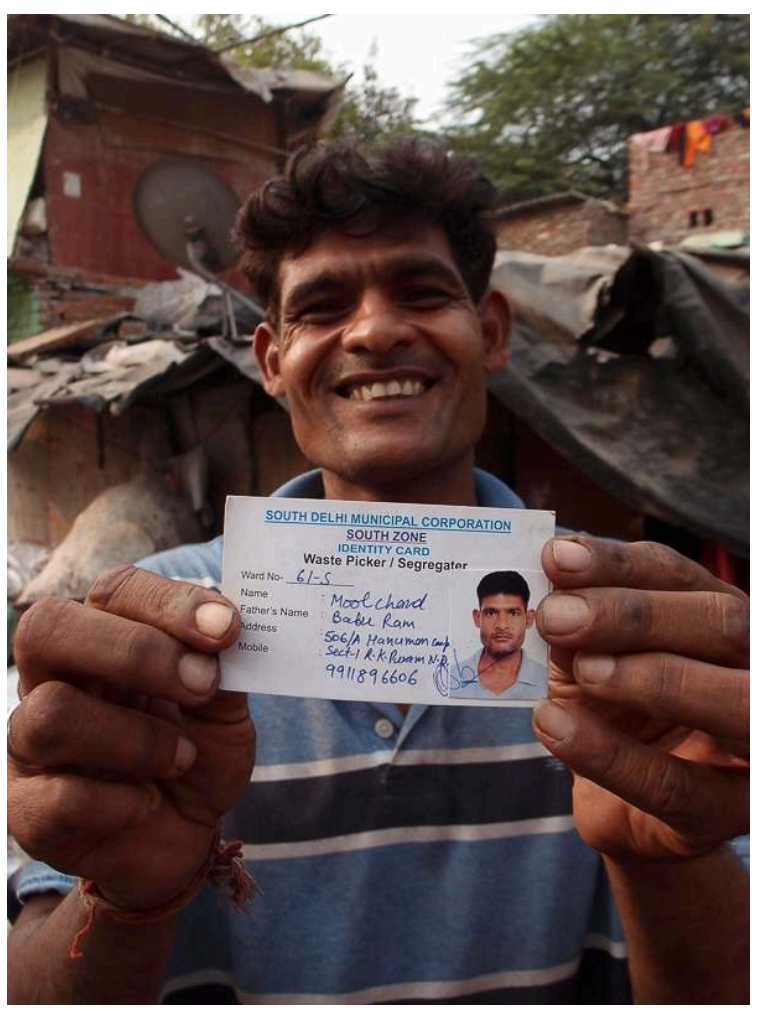

Moolchand est un waste picker qui vient d'Uttaranchal mais qui travaille à South Delhi depuis une quinzaine d'années. Je l'ai suivi à plusieurs reprises et je lui rends visite régulièrement lors de mes missions en Inde. Ce jour-là, il est heureux de me montrer sa carte d'identité de waste picker qu'il vient d'obtenir et qui lui assure une relative protection lors des contrôles policiers impromptus. Mais cela n'équivaut pas à une véritable formalisation de son travail : il n'est pas réellement intégré au service municipal, il reste à son compte et ne dispose d'aucun équipement fourni par la municipalité, ni de protection sociale ou de salaire alors qu'il contribue à nettoyer les rues des quartiers avoisinants. A l'image du logement sommaire que l'on devine derrière lui, Moolchand reste donc un travailleur précaire, en quête de reconnaissance.

Delhi, Inde, janvier 2016. Auteur : R. de Bercegol.

\section{Conclusion}

Longtemps écartés de la vue en raison de la contamination dont ils sont porteurs et de leur charge symbolique négative évoquant la souillure et le désordre, les déchets font aujourd'hui l'objet de processus de collecte et de requalification afin d'en extraire les matières valorisables avant leur mise au rebut définitive. Grâce à leurs composants - plastiques, métaux, papier, etc. - ils constituent une ressource qui produit désormais des bénéfices. Ce potentiel est d'autant plus essentiel que la raréfaction des ressources énergétiques et minérales implique une demande croissante en matières premières transformées et en produits recyclés.

La reconnaissance du rôle du secteur informel du recyclage apparaît primordiale pour en améliorer l'efficacité et créditer le travail réalisé par les récupérateurs. Les conditions de captage et de tri pourraient, en s'améliorant, augmenter les volumes des matériaux recyclés, réduisant, d'une part, le coût de gestion du transport et de l'élimination finale des déchets pour la collectivité et, d'autre part, rendant meilleures les conditions de travail tout en soutenant l'économie urbaine. Il s'agit, en effet, d'une 
ressource première pour de très nombreuses personnes qui en dépendent directement et qui sont impliquées aux différents niveaux, de la collecte au tri en passant par les multiples étapes de la transformation des matériaux. Cette économie de la récupération assure un revenu de base à des centaines de milliers de familles et alimente in fine en bout de chaîne les industries formelles de production de biens de consommations.

L'intégration du secteur ne peut se faire sans une reconnaissance des tâches accomplies par les récupérateurs en tant que "vrai travail», comme le rappellent dans les entretiens les personnes rencontrées ${ }^{5}$, utile socialement au même titre que d'autres métiers. Si cette activité est encore considérée illégale dans de nombreux pays, pour les récupérateurs la valeur économique, écologique et sociale de la récupération rend leur travail bénéfique pour la société et de ce fait digne : il contribue non seulement à une meilleure qualité de vie de ces travailleurs et de leurs familles sinon de tous les citadins. Tout se passe comme si la charge symbolique négative du contact avec la souillure, que les récupérateurs incorporent et souvent expriment par un sentiment de honte, serait soulagée grâce à la légitimation de leur activité. Les sentiments de n'être pas respecté ni reconnu fondent les revendications pour la reconnaissance (Honneth, 2000). Dans le cas des récupérateurs, cette demande est nourrie par la pénalisation (au sens juridique du terme) de l'activité et par les pénibles conditions de travail qui entraînent quelquefois des relations sociales de subordination, domination et exploitation.

Dépasser l'approche misérabiliste du secteur - certes, imparfait à bien des égards, mais dont, précisément, la relégation dans l'informalité favorise les abus - nous semble urgent afin de garantir son intégration à la gestion des déchets permettant ainsi d'assurer la mise en place progressive de normes sociales et environnementales. C'est, modestement, ce à quoi tend l'exposition La mise en image du rebut, qui élargit ce portfolio à d'autres terrains et à d'autres photos : présentée à des publics, à des publics divers (chercheurs, étudiants, professionnels des déchets, écoliers, grand public), sous différentes formes (vernissages, conférences, colloques, séminaires, tables-rondes) et en différents lieux ${ }^{6}$, elle cherche à sensibiliser et à engager le "spectateur " dans la réflexion sur nos rapports au déchet via le medium de l'image et contribuer en même temps à changer le regard sur ces travailleurs.

\section{BIBLIOGRAPHIE}

Chokhandre P., Singh S., Kashyap G. C., 2017. Prevalence, predictors and economic burden of morbidities among waste-pickers of Mumbai, India: a cross-sectional study. Journal of occupational medicine and toxicology[En ligne], vol. 12. doi : 10.1186/s12995-017-0176-3

Cirelli C., Florin B., (dir.), 2015. Sociétés urbaines et déchet. Éclairages internationaux, Presse Universitaire de Tours, Tours.

Corteel D., Le Lay S. (dir.), 2011, Les travailleurs des déchets. Toulouse, Érès, 336 p. 
Dagognet F., 2009. Éloge du déchet. In Beaune J.-C. (dir.), Le déchet, le rebut, le rien. Seyssel, Champ Vallon, p. 200-209

Friedmann D., 2010. Sociologie filmique, sociologie visuelle et écrit. La sociologie par l'image, Revue de l'Institut de Sociologie, $\mathrm{n}^{\circ} 11$.

Gill K. (dir.), 2012. Of Poverty and Plastic: Scavenging and Scrap Trading Entrepreneurs in India's Urban Informal Economy. New Delhi, Oxford University Press, 287 p.

Gunthert A. et al., 2007. La Fabrique des images contemporaines. Paris, Editions du Cercle d'art, 192 p. Honneth A., 2000 [1 1 ère ed. all. : 1992]. La lutte pour la reconnaissance. Paris, Les Éditions du Cerf, $232 \mathrm{p}$.

Ird-images, 2018. Recyclage au Vietnam, Dồng nát \& villages des métiers, film institutionnel. Réalisation : Boré J.M. - IRD IMAGES, 10'03min.

Jaligot R., Wilson D.C., Cheeseman C., Shaker B., 2016. Applying value chain analysis to informal sector recycling: a case study of Zabaleen. Resources, Conservation and Recycling, $n^{\circ} 114$, p. 80-91.

Jaglin S., Debout L., Salenson I., 2018. Du rebut à la ressource. Valorisation des déchets dans les villes du sud. Paris, Agence Française du Développement, 296 p. https://www.afd.fr/fr/du-rebut-laressource

Jeanjean A., 2006. Basses Euvres : une ethnologie du travail dans les égouts. Paris, Éd. du CTHS, 279 p.

Lhuilier D, Cochin Y., 1999. Des déchets et des hommes. Paris, Desclée de Brouwer, 184 p.

Louveau de la Guigneraye C., Arlaud J., 2007. De la photo au film : écritures numériques. Ethnologie française, vol. 37, p. 101-106.

Perelman M.D., 2007. Rebusque o trabajo? Un análisis a partir de las transformaciones del cirujeo en la Ciudad de Buenos Aires. In Suárez F., Schamber P. (coord.), Recicloscopio. Miradas sobre recuperadores urbanos de residuos en América Latina. Buenos Aires, Universidad Nacional General Sarmiento, Universidad Nacional de Lanús, Prometeo Libros.

Scheinberg A., Spies S., Simpson M.H., et al., 2011. Assessing urban recycling in low- and middle income countries: Building on modernized mixtures. Habitat International, n 35, p. 188-198.

Scheinberg A., Simpson M., 2015. A tale of five cities: using recycling frameworks to analyse inclusive recycling performances. Waste Management Resources, $n^{\circ} 33$, p. 975-985.

Scheinberg A., Nesic J., Savain R.A., et al., 2016. From collision to collaboration: Integrating informal recyclers and re-use operators in Europe, a review. Waste Management \& Research, vol. 34, $\mathrm{n}^{\circ}$ 9, p. 820-839.

Sontag S., 1977. On Photography. Penguin Books, London, 224 p.

Suárez F., Schamber P., (coord.), 2007. Recicloscopio. Miradas sobre recuperadores urbanos de residuos en América Latina. Universidad Nacional General Sarmiento, Universidad Nacional de Lanús, Prometeo Libros, Buenos Aires.

\section{NOTES}

1. https://sud.hypotheses.org/1940

2. Sociétés urbaines et déchets (SUD) émane de l'axe transversal de recherche éponyme porté depuis 2009 par Claudia Cirelli et Bénédicte Florin, respectivement membre des équipes CoST et EMAM de l'UMR 7324 CITERES (CNRS, Université de Tours). À partir d'approches 
pluridisciplinaires et internationales, les travaux des membres de SUD ont l'ambition de saisir les recompositions et les tensions à l'œuvre dans le domaine de la gestion des déchets dans les villes. À la suite de plusieurs séminaires de recherche, rencontres et actions collectives, SUD s'est étoffé et regroupe aujourd'hui plus d'une quarantaine de chercheurs ou professionnels des déchets. Les activités de SUD s'appuient également sur un carnet de recherche du même nom: https:// sud.hypotheses.org

3. Pour des raisons éditoriales, ce travail méthodologique (une fiche de renseignements par image sélectionnée) n'est pas présenté dans le présent portfolio.

4. La question du travail informel autour des déchets n'est souvent abordée que du côté des "pays du Sud", alors même que nous pouvons tous croiser en France des personnes qui collectent ainsi des rebuts pour les revendre afin de gagner leur vie. L'article Faire la ferraille en banlieue parisienne publié dans ce même numéro d'EchoGéo en donne plus d'un exemple (Florin et Garret, 2019).

5. Les recherches sur l'expérience des individus engagés dans la récupération après une perte d'emploi montrent combien cette revendication est particulièrement importante (Perelman, 2007).

6. Des variations locales, adaptées au contexte, ont été proposées comme, par exemple, à Delhi lors de l'événement City of Waste (organisé par Rémi de Bercegol, Christine, Ithurbide et François Grosjean à l'Indian Habitat Centre, au métro Mandi House et à l'université de Jamia Millia Islamia, de février à octobre 2018) lors duquel l'exposition a été enrichie par de nombreuses photographies prises sur le terrain indien. Le réseau SUD se tient à la disposition des institutions intéressées par l'exposition, mais aussi ouvert aux propositions de collaborations de recherche. Contacts : https://sud.hypotheses.org/credits

\section{AUTEURS}

\section{CLAUDIA CIRELLI}

Claudia Cirelli, claudia.cirelli@univ-tours.fr, est chercheure associée de l’UMR CITERES

(Université de Tours). Elle a publié récemment :

- Cirelli C., Rosa É., 2018. Scavenging: Between precariousness, marginality and access to the city. The case of Roma people in Turin and Marseille. Environment \& Planning A, vol. 50, $\mathrm{n}^{\circ} 7$, p. 1407-1424.

- Cirelli C. et al., 2017. "L'incinérateur est trop près, la poubelle trop loin" : gérer les déchets en régime de proximité. Flux, n 109-110, p. 61-72.

- Cirelli C., Florin B., (dir.), 2015. Sociétés urbaines et déchets. Éclairages internationaux. Tours, Presse Universitaire de Tours, $452 \mathrm{p}$.

\section{BÉNÉDICTE FLORIN}

Bénédicte Florin, benedicte.florin@univ-tours.fr, maître de conférences à l'Université de Tours et membre de l'UMR CITERES, équipe Monde Arabe et Musulman (EMAM). Elle a publié récemment : - Florin B., 2018. The Rag Pickers of Cairo: The Dregs of the City or "Garbage Businessmen"? Ethnologie française, $\mathrm{n}^{\circ} 153$, p. 487-498.

- Florin B., 2016. De l'indignité à l'indignation : petites luttes, résistances quotidiennes et 
tentatives de mobilisation des récupérateurs de déchets à Istanbul. Cultures \& conflits [En ligne], $\mathrm{n}^{\circ}$ 101, p. 99-120. http://journals.openedition.org/conflits/19184

- Allix É., Florin B., 2016. Indésirables dans la ville, utiles dans l'ordure. Les récupérateurs de déchets au Maroc. Géographie et culture [En ligne], n 96, p. 23-45. http://

journals.openedition.org/gc/4434

\section{RÉMI DE BERCEGOL}

Rémi de Bercegol, remi.debercegol@cnrs.fr, est chargé de recherche au Centre National de la Recherche Scientifique, membre de l'UMR PRODIG (Paris). Il a co-organisé à Delhi l'exposition The City of Waste, version anglaise de l'expo dont sont extraites les photos. Il a publié récemment :

- de Bercegol R., 2019 [1ère ed. 2010]. Small Towns and Decentralisation in India. Rawat Publications [1 1 ère ed. Springer], $246 \mathrm{p}$.

- de Bercegol R., Gowda S., 2018. Waste and Energy Nexus: Rethinking the modernisation of waste services in Delhi. Urban Studies, Special Issue on 'Urban Nexus', June 2018.

- de Bercegol R., 2018. Les enjeux de la valorisation des déchets à Delhi. In Jaglin S, Debout L., Salenson I., (dir.), Du rebut à la ressource. Valorisation des déchets dans les villes du sud. Paris, AFD, p. 31-49. 